\title{
Coupled finite element - hierarchical boundary element methods for dynamic soil-structure interaction in the frequency domain
}

\author{
P. Coulier*, S. François, G. Lombaert, G. Degrande \\ KU Leuven, Department of Civil Engineering, Kasteelpark Arenberg 40, B-3001 Leuven, Belgium
}

\begin{abstract}
SUMMARY
This paper discusses the coupling of finite element and fast boundary element methods for the solution of dynamic soil-structure interaction problems in the frequency domain. The application of hierarchical matrices in the boundary element formulation allows considering much larger problems compared to classical methods. Three coupling methodologies are presented and their computational performance is assessed through numerical examples. It is demonstrated that the use of hierarchical matrices renders a direct coupling approach the least efficient, as it requires the assembly of a dynamic soil stiffness matrix. Iterative solution procedures are presented as well, and it is shown that the application of such schemes to dynamic soil-structure interaction problems in the frequency domain is not trivial, as convergence can hardly be achieved if no relaxation procedure is incorporated. Aitken's $\Delta^{2}$-method is therefore employed in sequential iterative schemes for the calculation of an optimized interface relaxation parameter, while a novel relaxation technique is proposed for parallel iterative algorithms. It is demonstrated that the efficiency of these algorithms strongly depends on the boundary conditions applied to each subdomain; the fastest convergence is observed if Neumann boundary conditions are imposed on the stiffest subdomain. The use of a dedicated solver for each subdomain hence results in a reduced computational effort. A monolithic coupling strategy, often used for the solution of fluid-structure interaction problems, is also introduced. The governing equations are simultaneously solved in this approach, while the assembly of a dynamic soil stiffness matrix is avoided.

Copyright (c) 2014 John Wiley \& Sons, Ltd.
\end{abstract}

Received ...

KEY WORDS: Dynamic soil-structure interaction; FE-BE coupling; iterative methods; interface relaxation; $\mathscr{H}$-matrices; visco-elastodynamics.

\section{INTRODUCTION}

The numerical solution of three-dimensional (3D) dynamic soil-structure interaction (SSI) problems is a challenging task [1]. A domain decomposition approach is often introduced in numerical models, allowing for the application of different numerical techniques for the soil and the structure. The coupled finite element - boundary element (FE-BE) method is a well-known approach, in which the FE method allows to model structures with complex geometries while the $\mathrm{BE}$ method enables accounting for the radiation of waves in domains of (semi-)infinite extent. The complementarity of both methods can either be exploited in the time [2] or in the frequency domain [3].

In the past decades, a lot of attention has been paid to the development of efficient algorithms for the coupling of FE and BE models $[4,5,6]$. Direct and iterative coupling methodologies are generally distinguished. Furthermore, a distinction between conforming or non-conforming interface discretizations is made, where the coupling conditions are either imposed in a strong or

*Correspondence to: P. Coulier; Fax: +32 1632 19 88; E-mail pieter.coulier@bwk.kuleuven.be 
weak sense. The latter allow for independent mesh sizes for each subdomain. FE-BE coupling algorithms for elastostatics are discussed, among others, by Elleithy et al. [7] and Margonari et al. [8], while Rüberg et al. [9] present an algorithm for time domain elastodynamics using nonconforming interfaces where the coupling conditions are incorporated in a weak sense by means of Lagrange multipliers.

The applicability of classical BE formulations to large scale problems is hindered by stringent memory and CPU requirements resulting from dense, fully populated unsymmetric matrices. This has led to the development of fast BE methods to improve the computational efficiency, including the fast multipole method (FMM) [10] and methods based on hierarchical matrices ( $\mathscr{H}$-matrices) [11], which allow increasing the problem size compared to classical BE formulations. Recently, a $\mathscr{H}$-BE method for visco-elastodynamics in the frequency domain incorporating Green's functions for a horizontally layered halfspace has been presented [12]. These Green's functions are computed by means of the direct stiffness method [13,14], as no closed form analytical expressions are available; their application avoids meshing of the free surface and layer interfaces to model wave propagation in a stratified medium.

The application of $\mathscr{H}$-matrices in BE formulations affects the efficiency of FE-BE coupling algorithms. This paper therefore aims to present suitable $\mathrm{FE}-\mathscr{H}$-BE coupling procedures for the solution of dynamic SSI problems and to compare their computational performance. Throughout this paper, non-overlapping domains with conforming interface meshes are considered and all methods are formulated in the frequency domain. Three different FE- $\mathscr{H}$-BE coupling methodologies are discussed. First, a classical direct coupling strategy is introduced, which requires the assembly of a dynamic soil stiffness matrix to obtain a global set of coupled equations. Next, iterative algorithms are presented; the governing equations are solved separately for each subdomain, while the boundary conditions at the soil-structure interface are updated until convergence is achieved. Sequential (Neumann-Dirichlet, Dirichlet-Neumann) as well as parallel (Neumann-Neumann, Dirichlet-Dirichlet) algorithms are considered. The application of iterative schemes to dynamic SSI problems in the frequency domain has only received limited attention in the literature so far [15], as it is not easy to achieve convergence with these algorithms [16]. Special attention is therefore paid to optimized interface relaxation techniques in order to ensure and/or speed up the convergence. For the sequential algorithms, Aitken's $\Delta^{2}$-method [17] is employed, while a novel relaxation technique is presented for the parallel iterative schemes. Finally, a monolithic coupling approach is proposed, in which the governing equations of both subdomains are solved simultaneously, while the assembly of a dynamic soil stiffness matrix is avoided. Monolithic coupling schemes are often used to solve fluid-structure interaction problems [18, 19], but their application to dynamic SSI problems is not common.

The text is organized as follows. Section 2 briefly summarizes the governing equations of the FE and $\mathscr{H}$-BE method. Three FE- $\mathscr{H}$-BE coupling procedures are subsequently introduced in section 3. Numerical examples are investigated in section 4 in order to verify these coupling strategies and to assess their computational performance. This allows for the formulation of guidelines concerning the choice of an appropriate coupling strategy for a specific dynamic SSI problem. The applicability of coupled FE- $\mathscr{H}$-BE methods to large scale problems is finally demonstrated in section 5, where the wave impeding effect of a block of stiffened soil of finite length embedded in a halfspace is investigated.

\section{FINITE ELEMENT AND BOUNDARY ELEMENT METHODS}

The governing equations of the FE and $\mathscr{H}$-BE method are summarized in this section. It is assumed that finite elements are used to model the structural domain $\Omega_{\mathrm{b}}$, while boundary elements are employed to model wave propagation in the surrounding soil domain $\Omega_{\mathrm{s}}$. The domain $\Omega_{\mathrm{b}}$ represents a generalized structure, comprising the actual structure and part of the soil domain, as depicted in figure 1. The soil-structure interface is denoted as $\Sigma$. 


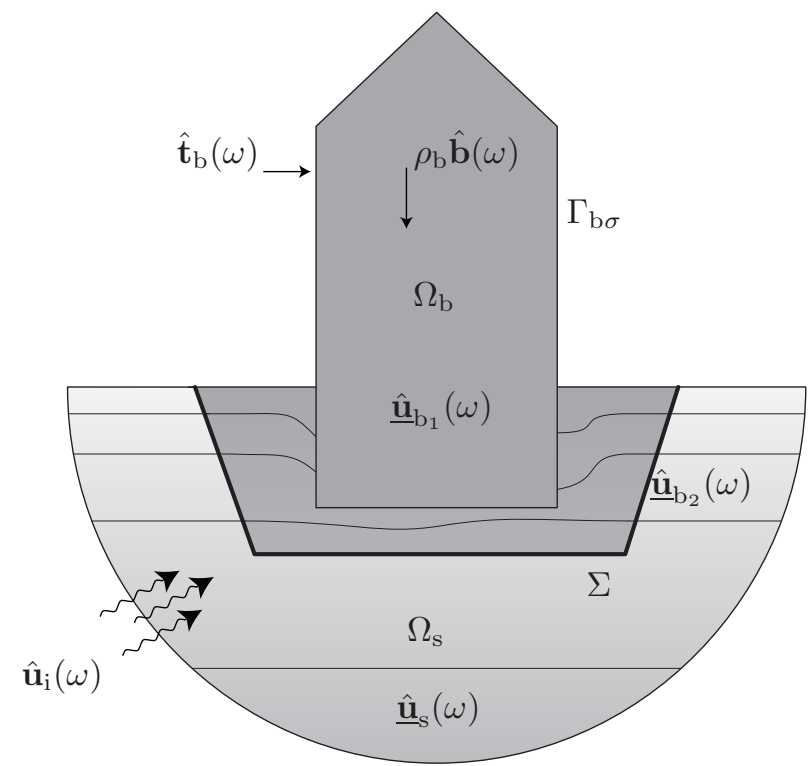

Figure 1. Geometry of the subdomains: structural domain $\Omega_{\mathrm{b}}$ and soil domain $\Omega_{\mathrm{s}}$.

\subsection{Finite element formulation}

Finite element equations of the domain $\Omega_{\mathrm{b}}$ are obtained by introducing a FE discretization in the weak variational formulation of the equilibrium equations of $\Omega_{\mathrm{b}}$ (based on the principle of virtual work) and subsequently applying a Galerkin procedure. This provides the following set of equations [20]:

$$
\left[\mathbf{K}_{\mathrm{b}}+i \omega \mathbf{C}_{\mathrm{b}}-\omega^{2} \mathbf{M}_{\mathrm{b}}\right] \underline{\hat{\mathbf{u}}}_{\mathrm{b}}(\omega)=\underline{\hat{\mathbf{f}}}_{\mathrm{b}}(\omega)+\underline{\hat{\mathbf{f}}}_{\mathrm{b}}^{\mathrm{s}}(\omega)
$$

where a hat above a variable denotes its representation in the frequency domain. $\underline{\hat{\underline{u}}}_{\mathrm{b}}(\omega)$ collects the nodal degrees of freedom, while $\mathbf{K}_{\mathrm{b}}, \mathbf{C}_{\mathrm{b}}$, and $\mathbf{M}_{\mathrm{b}}$ are the stiffness, damping, and mass matrices, respectively. The bracketed term on the left hand side of equation (1) is identified as the dynamic stiffness matrix $\widehat{\mathbf{K}}_{\mathrm{b}}(\omega)=\mathbf{K}_{\mathrm{b}}+i \omega \mathbf{C}_{\mathrm{b}}-\omega^{2} \mathbf{M}_{\mathrm{b}}$ of the structure. The force vector $\underline{\hat{\mathbf{f}}}_{\mathrm{b}}(\omega)$ results from the body forces $\rho_{\mathrm{b}} \hat{\mathbf{b}}(\omega)$ on $\Omega_{\mathrm{b}}$ and the tractions $\hat{\mathbf{t}}_{\mathrm{b}}(\omega)$ on the boundary $\Gamma_{\mathrm{b} \sigma}$, while $\hat{\underline{\mathbf{f}}}_{\mathrm{b}}^{\mathrm{s}}(\omega)$ is due to the incident wavefield $\hat{\mathbf{u}}_{\mathrm{i}}(\omega)$ on $\Sigma$ (figure 1). Adequate solvers which account for the sparsity and symmetry of the system can be employed to solve equation (1).

\subsection{Hierarchical boundary element formulation}

The BE method is based on the discretization of the boundary $\Sigma$ of a domain $\Omega_{\mathrm{s}}$ with an appropriate number of boundary elements in order to numerically solve a boundary integral equation [21]. Throughout this paper, a regularized boundary integral equation is employed, in which the evaluation of Cauchy principal value (CPV) integrals is avoided [21, 22, 23]. The regularization procedure is based on the fact that the singularity of the static and dynamic Green's functions at the source point is similar. For an unbounded domain $\Omega_{\mathrm{s}}$, the displacements $\underline{\hat{\mathbf{u}}}(\omega)$ and tractions $\underline{\hat{\mathbf{t}}}(\omega)$ at the collocation points of the boundary $\Sigma$ are related as follows:

$$
[\widehat{\mathbf{T}}(\omega)+\mathbf{I}] \underline{\hat{\mathbf{u}}}(\omega)=\widehat{\mathbf{U}}(\omega) \underline{\underline{\mathbf{t}}}(\omega)
$$

where $\widehat{\mathbf{T}}(\omega)$ and $\widehat{\mathbf{U}}(\omega)$ are BE collocation matrices, while I represents a unit matrix, corresponding to the integral free term in the boundary integral equation. The latter vanishes for a bounded domain. The computation of $\widehat{\mathbf{T}}(\omega)$ and $\widehat{\mathbf{U}}(\omega)$ requires integration of the Green's tractions and displacements over the boundary $\Sigma$, respectively. The integral representation theorem allows for the computation 
of the radiated wavefield in the soil $\underline{\underline{\underline{u}}}_{\mathrm{s}}(\omega)$ from the displacements and tractions on $\Sigma$. In order to mitigate the occurrence of fictitious eigenfrequencies in the application of the BE method to external wave propagation problems, the Combined Helmholtz Integral Equation Formulation (CHIEF) proposed by Schenk [24] is employed.

The BE method leads to a reduction of the spatial problem dimension (i.e. surface instead of volume discretization), but the storage of the fully populated matrices $\widehat{\mathbf{T}}(\omega)$ and $\widehat{\mathbf{U}}(\omega)$ requires a quadratic amount of memory with respect to the number of degrees of freedom $N_{\text {DOF }}$, while a cubic amount of numerical operations is needed to solve the corresponding equation (2) by means of direct numerical solvers. The use of $\mathscr{H}$-matrices provides an elegant way to treat fully populated matrices with almost linear complexity [25], as they approximate the original matrices (with an arbitrary prescribed accuracy) by means of memory efficient representations. The reader is referred to the literature $[11,25,26]$ for a comprehensive overview of fast $\mathrm{BE}$ methods based on $\mathscr{H}$-matrices. The construction of $\mathscr{H}$-matrices is based on the identification of admissible and inadmissible hierarchical cluster pairs in the BE mesh; the partially pivoted adaptive cross approximation (ACA) algorithm $[27,28]$ is employed to compute low rank approximations of matrix blocks corresponding to admissible cluster pairs. A major advantage of ACA is its purely algebraic character, avoiding the need for (semi-)analytical expressions of the Green's functions employed in the $\mathrm{BE}$ formulation, which enables its application to problems involving elastodynamic wave propagation in anisotropic [29] or layered [12] media. All BE calculations in the remainder of this paper involve the application of $\mathscr{H}$-matrices.

Assembling hierarchical approximations $\widehat{\mathbf{T}}_{\mathscr{H}}(\omega)$ and $\widehat{\mathbf{U}}_{\mathscr{H}}(\omega)$ of the BE collocation matrices $\widehat{\mathbf{T}}(\omega)$ and $\widehat{\mathbf{U}}(\omega)$, respectively, allows replacing the BE equation (2) by:

$$
\left[\widehat{\mathbf{T}}_{\mathscr{H}}(\omega)+\mathbf{I}\right] \underline{\hat{\mathbf{u}}}(\omega)=\widehat{\mathbf{U}}_{\mathscr{H}}(\omega) \underline{\hat{\mathbf{t}}}(\omega)
$$

Equation (3) can be rewritten as:

$$
\widehat{\mathbf{A}}_{\mathscr{H}}(\omega) \underline{\hat{\mathbf{x}}}(\omega)=\underline{\hat{\mathbf{b}}}(\omega)
$$

where the vector of unknowns $\underline{\hat{\mathbf{x}}}(\omega)$ contains displacements, tractions or both, depending on whether a Neumann, Dirichlet or mixed Neumann-Dirichlet problem is considered. In order to solve equation (4), iterative Krylov subspace methods such as the generalized minimal residual method (GMRES) [30] are well suited. The matrix-vector multiplication forms the core of iterative solvers, and the complexity of this operation is only $\mathcal{O}\left(N_{\text {DOF }} \log N_{\text {DOF }}\right)$ for $\mathscr{H}$-matrices [26]. A tolerance of $10^{-6}$ is adopted in the iterative solver for the relative residual norm $\| \underline{\hat{b}}(\omega)-$ $\widehat{\mathbf{A}}_{\mathscr{H}}(\omega) \underline{\hat{\mathbf{x}}}(\omega)\|/\| \underline{\hat{\mathbf{b}}}(\omega) \|$ in all numerical examples presented in this paper.

As will be clarified in section 3, equation (4) has to be solved for multiple right hand sides $\underline{\hat{b}}(\omega)$ in $\mathrm{FE}-\mathscr{H}$-BE coupling algorithms; the implementation of a suitable preconditioner is therefore desirable to reduce the computation time. A right preconditioner $\widehat{\mathbf{M}}(\omega)$ is used throughout this paper in order to lower the condition number of the coefficient matrix $\widehat{\mathbf{A}}_{\mathscr{H}}(\omega)$ :

$$
\widehat{\mathbf{A}}_{\mathscr{H}}(\omega) \widehat{\mathbf{M}}^{-1}(\omega) \underline{\hat{\mathbf{y}}}(\omega)=\underline{\hat{\mathbf{b}}}(\omega)
$$

with $\widehat{\mathbf{M}}(\omega) \underline{\hat{\mathbf{x}}}(\omega)=\hat{\mathbf{y}}(\omega)$. An example of an efficient preconditioner is the approximate $\mathscr{H}-\mathrm{LU}$ decomposition [31]; its computation requires, however, additional arithmetic operations. A much simpler strategy is applied in the present paper, following the approach recently adopted by Chaillat et al. [32] for the acceleration of the fast multipole method for elastodynamics. A block diagonal preconditioner $\widehat{\mathbf{M}}(\omega)=\operatorname{blkdiag}\left(\widehat{\mathbf{A}}_{\mathscr{H}}(\omega)\right)$ is employed, where the size of the diagonal blocks is determined by the lowest hierarchical cluster level. An inner GMRES solver with a moderate tolerance of $10^{-2}$ is applied to solve the preconditioning linear systems, resulting in a nested innerouter iteration scheme. Furthermore, the flexible GMRES (FGMRES) algorithm [33] is employed for the outer iteration in order to avoid the explicit multiplication of $\widehat{M}^{-1}(\omega)$ with the Krylov vectors. As $\widehat{\mathbf{M}}(\omega)$ is already computed and stored, the proposed approach is very cheap in terms of computational resources. 


\section{FE- $\mathscr{H}$-BE COUPLING PROCEDURES}

Three procedures for the coupling of FE and $\mathscr{H}$-BE models are outlined in this section. The numerical verification and the assessment of the computational efficiency of these methods are subsequently addressed in section 4 .

\subsection{Direct FE- $\mathscr{H}-B E$ coupling}

In a classical direct coupling strategy [34], the governing equations of the FE and BE subdomain are straightforwardly combined, accounting for continuity of displacements and equilibrium of tractions at the soil-structure interface $\Sigma$. This results in a global coupled system of equations:

$$
\left(\left[\begin{array}{ll}
\widehat{\mathbf{K}}_{\mathrm{b}_{1} \mathrm{~b}_{1}}(\omega) & \widehat{\mathbf{K}}_{\mathrm{b}_{1} \mathrm{~b}_{2}}(\omega) \\
\widehat{\mathbf{K}}_{\mathrm{b}_{2} \mathrm{~b}_{1}}(\omega) & \widehat{\mathbf{K}}_{\mathrm{b}_{2} \mathrm{~b}_{2}}(\omega)
\end{array}\right]+\left[\begin{array}{cc}
\mathbf{0} & \mathbf{0} \\
\mathbf{0} & \widehat{\mathbf{K}}_{\mathrm{b}_{2} \mathrm{~b}_{2}}^{\mathrm{s}}(\omega)
\end{array}\right]\right)\left\{\begin{array}{l}
\hat{\underline{\hat{\mathbf{u}}}}_{\mathrm{b}_{1}}(\omega) \\
\underline{\underline{\mathbf{u}}}_{\mathrm{b}_{2}}(\omega)
\end{array}\right\}=\left\{\begin{array}{l}
\hat{\mathbf{f}}_{\mathrm{b}_{1}}(\omega) \\
\underline{\hat{\mathbf{f}}}_{\mathrm{b}_{2}}(\omega)
\end{array}\right\}+\left\{\begin{array}{c}
\underline{\mathbf{0}} \\
\underline{\hat{\mathbf{f}}}_{\mathrm{b}_{2}}^{\mathrm{s}}(\omega)
\end{array}\right\}
$$

where a subdivision into block matrices according to internal degrees of freedom $\underline{\hat{\underline{u}}}_{\mathrm{b}_{1}}(\omega)$ in the structural domain $\Omega_{\mathrm{b}}$ and degrees of freedom $\underline{\hat{\mathbf{u}}}_{\mathrm{b}_{2}}(\omega)$ on the soil-structure interface $\Sigma$ is introduced (figure 1). The corresponding number of degrees of freedom are indicated as $n_{\text {dof }_{1}}$ and $n_{\text {dof }_{2}}$, respectively. $\widehat{\mathbf{K}}_{\mathrm{b}_{2} \mathrm{~b}_{2}}^{\mathrm{s}}(\omega)$ represents the dynamic soil stiffness matrix and is defined as:

$$
\widehat{\mathbf{K}}_{\mathrm{b}_{2} \mathrm{~b}_{2}}^{\mathrm{s}}(\omega)=\int_{\Sigma} \mathbf{N}_{\mathrm{b}_{2}}^{\mathrm{T}}(\mathbf{x}) \mathbf{N}_{\mathrm{b}_{2}}(\mathbf{x}) \underline{\hat{\mathbf{t}}}\left(\mathbf{N}_{\mathrm{b}_{2}}(\mathbf{x})\right)(\omega) \mathrm{d} S=\mathbf{T}_{\mathrm{q}} \hat{\hat{\mathbf{t}}}\left(\mathbf{N}_{\mathrm{b}_{2}}(\mathbf{x})\right)(\omega)
$$

where $\mathbf{N}_{\mathrm{b}_{2}}(\mathrm{x})$ indicates the FE shape functions on the soil-structure interface $\Sigma$, conforming with the BE interpolation functions. The frequency independent matrix $\mathbf{T}_{\mathrm{q}}=\int_{\Sigma} \mathbf{N}_{\mathrm{b}_{2}}^{\mathrm{T}}(\mathbf{x}) \mathbf{N}_{\mathrm{b}_{2}}(\mathbf{x}) \mathrm{d} S$ links the FE and BE discretizations. Both $\widehat{\mathbf{K}}_{\mathrm{b}_{2} \mathrm{~b}_{2}}^{\mathrm{s}}(\omega)$ and $\mathbf{T}_{\mathrm{q}}$ have dimensions $\left(n_{\mathrm{dof}_{2}} \times n_{\mathrm{dof}_{2}}\right)$.

Although equation (6) provides a straightforward solution to the dynamic SSI problem, it suffers some major drawbacks. Equation (7) requires the evaluation of tractions $\underline{\hat{\mathbf{t}}}\left(\mathbf{N}_{\mathrm{b}_{2}}(\mathbf{x})\right)(\omega)$ by means of the $\mathscr{H}$-BE method, which requires the solution of equation (5) for all shape functions $\mathbf{N}_{\mathrm{b}_{2}}(\mathbf{x})$ on $\Sigma$; the implemented FGMRES algorithm is only able to handle one right hand side at a time. Furthermore, addition of the dense unsymmetric dynamic soil stiffness matrix $\widehat{\mathbf{K}}_{\mathrm{b}_{2} \mathrm{~b}_{2}}^{\mathrm{s}}(\omega)$ to the dynamic stiffness matrix of the structure strongly affects the sparsity of the system, reducing the efficiency of sparse FE solvers. The numerical examples in section 4 will demonstrate that this conventional approach, in which the dynamic soil stiffness matrix $\widehat{\mathbf{K}}_{\mathrm{b}_{2} \mathrm{~b}_{2}}^{\mathrm{s}}(\omega)$ is explicitly evaluated, does not provide an efficient solution procedure, especially for large problems.

The computational effort can be limited by considering a reduced kinematic basis for the displacement vector $\underline{\underline{\hat{u}}}_{\mathrm{b}_{2}}(\omega)$ on the interface $\Sigma$ through the introduction of a modal decomposition $\underline{\hat{\underline{u}}}_{\mathrm{b}_{2}}(\omega) \simeq \boldsymbol{\Psi}_{\mathrm{b}_{2}} \underline{\hat{\alpha}}(\omega)$, where $\boldsymbol{\Psi}_{\mathrm{b}_{2}}$ and $\underline{\hat{\alpha}}(\omega)$ collect the mode shapes and the modal coordinates, respectively [35]. This allows rewriting equation (6) in terms of modal coordinates $\underline{\hat{\alpha}}(\omega)$, and only a modal soil stiffness matrix $\boldsymbol{\Psi}_{\mathrm{b}_{2}}^{\mathrm{T}} \widehat{\mathbf{K}}_{\mathrm{b}_{2} \mathrm{~b}_{2}}^{\mathrm{s}}(\omega) \boldsymbol{\Psi}_{\mathrm{b}_{2}}$ with dimensions $\left(n_{\mathrm{m}_{2}} \times n_{\mathrm{m}_{2}}\right)$ has to be computed:

$$
\boldsymbol{\Psi}_{\mathrm{b}_{2}}^{\mathrm{T}} \widehat{\mathbf{K}}_{\mathrm{b}_{2} \mathrm{~b}_{2}}^{\mathrm{s}}(\omega) \boldsymbol{\Psi}_{\mathrm{b}_{2}}=\int_{\Sigma}\left(\mathbf{N}_{\mathrm{b}_{2}}(\mathbf{x}) \boldsymbol{\Psi}_{\mathrm{b}_{2}}\right)^{\mathrm{T}} \mathbf{N}_{\mathrm{b}_{2}}(\mathbf{x}) \underline{\hat{\mathbf{t}}}\left(\mathbf{N}_{\mathrm{b}_{2}}(\mathbf{x}) \boldsymbol{\Psi}_{\mathrm{b}_{2}}\right)(\omega) \mathrm{d} S
$$

As a result, equation (5) is only solved $n_{\mathrm{m}_{2}}$ times for tractions $\underline{\hat{\mathrm{t}}}\left(\mathbf{N}_{\mathrm{b}_{2}}(\mathbf{x}) \boldsymbol{\Psi}_{\mathrm{b}_{2}}\right)(\omega)$; the number of modes $n_{\mathrm{m}_{2}}$ is generally much lower than the number of degrees of freedom $n_{\mathrm{dof}_{2}}$ on the interface $\Sigma$. Although a substantial reduction in computation time can be achieved compared to the use of a full kinematic basis, the resulting modal soil stiffness matrix $\boldsymbol{\Psi}_{\mathrm{b}_{2}}^{\mathrm{T}} \widehat{\mathbf{K}}_{\mathrm{b}_{2} \mathrm{~b}_{2}}^{\mathrm{s}}(\omega) \boldsymbol{\Psi}_{\mathrm{b}_{2}}$ remains dense and unsymmetric, consequently affecting the efficiency of sparse FE solvers. Furthermore, an appropriate choice of the modes $\Psi_{\mathrm{b}_{2}}$ is required to obtain accurate results.

\subsection{Iterative $F E-\mathscr{H}-B E$ coupling}

Iterative coupling procedures provide a valuable alternative to the conventional direct strategy outlined in the previous subsection. The governing equations are solved separately for each 
subdomain, while the boundary conditions at the soil-structure interface are updated until convergence is achieved. This methodology avoids the assembly and solution of a global system of coupled equations; it hence allows for the independent use of dedicated FE and $\mathscr{H}$-BE solvers in both subdomains. Iterative schemes are often used for dynamic SSI problems in the time domain to allow for the use of different time discretization schemes in the FE and BE subdomains [2, 36, 37]. Their application in the frequency domain remains rather limited, however, especially due to convergence difficulties [16]. Frequency domain iterative algorithms described in the literature mainly involve acoustic-acoustic [38] and acoustic-elastodynamic [16] coupling; the iterative coupling of FE and fast multipole BE models for visco-elastodynamics in the frequency domain is discussed by Grasso [15].

Four different iterative algorithms for the coupling of FE and $\mathscr{H}$-BE models are outlined in the following subsections. These methodologies are denoted as sequential Neumann-Dirichlet or Dirichlet-Neumann algorithms, and parallel Neumann-Neumann or Dirichlet-Dirichlet algorithms, indicating which kind of boundary conditions are imposed on the FE and BE subdomain, respectively.

\subsubsection{Sequential Neumann-Dirichlet algorithm}

At iteration step $k$ of the sequential Neumann-Dirichlet procedure, the finite element subdomain is analyzed with Neumann boundary conditions $\underline{\hat{\mathbf{q}}}^{k}(\omega)$ at the soil-structure interface $\Sigma$ :

$$
\left[\begin{array}{ll}
\widehat{\mathbf{K}}_{\mathrm{b}_{1} \mathrm{~b}_{1}}(\omega) & \widehat{\mathbf{K}}_{\mathrm{b}_{1} \mathrm{~b}_{2}}(\omega) \\
\widehat{\mathbf{K}}_{\mathrm{b}_{2} \mathrm{~b}_{1}}(\omega) & \widehat{\mathbf{K}}_{\mathrm{b}_{\mathrm{b}_{2}}(\omega)}(\omega)
\end{array}\right]\left\{\begin{array}{l}
\hat{\mathbf{u}}_{\mathrm{b}_{1}}^{k}(\omega) \\
\underline{\hat{\mathbf{u}}}_{\mathrm{b}_{2}}^{k}(\omega)
\end{array}\right\}=\left\{\begin{array}{l}
\hat{\hat{\mathbf{f}}}_{\mathrm{b}_{1}}(\omega) \\
\underline{\hat{\mathbf{f}}}_{\mathrm{b}_{2}}(\omega)
\end{array}\right\}+\left\{\begin{array}{c}
\underline{\mathbf{0}} \\
\underline{\hat{\mathbf{f}}}_{\mathrm{b}_{2}}^{\mathrm{s}}(\omega)
\end{array}\right\}+\left\{\begin{array}{c}
\underline{\mathbf{0}} \\
\underline{\hat{\mathbf{q}}}^{k}(\omega)
\end{array}\right\}
$$

where $\underline{\hat{\mathbf{q}}}^{k}(\omega)$ denotes the soil-structure interaction forces. Solving equation (9) by means of a standard finite element solver provides the internal and interface displacements $\underline{\hat{\underline{u}}}_{\mathrm{b}_{1}}^{k}(\omega)$ and $\underline{\hat{\underline{u}}}_{\mathrm{b}_{2}}^{k}(\omega)$. The latter are subsequently imposed as Dirichlet boundary conditions on the boundary element subdomain, allowing to solve the preconditioned equation (5) for the interface tractions $\underline{\hat{t}}^{k}(\omega)$ using the FGMRES solver. These tractions are used to calculate equivalent nodal forces $\underline{\hat{\hat{q}}}^{k+1}(\omega)$ :

$$
\underline{\hat{\mathbf{q}}}^{k+1}(\omega)=-\int_{\Sigma} \mathbf{N}_{\mathrm{b}_{2}}^{\mathrm{T}}(\mathbf{x}) \mathbf{N}_{\mathrm{b}_{2}}(\mathbf{x}) \hat{\mathbf{t}}^{k}(\omega) \mathrm{d} S=-\mathbf{T}_{\mathrm{q}} \underline{\hat{\mathbf{t}}}^{k}(\omega)
$$

where a tilde above a variable indicates an unrelaxed quantity. The interaction forces are finally relaxed using an iteration dependent relaxation parameter $\lambda^{k}$ :

$$
\underline{\hat{\mathbf{q}}}^{k+1}(\omega)=\lambda^{k} \underline{\hat{\mathbf{q}}}^{k+1}(\omega)+\left(1-\lambda^{k}\right) \underline{\hat{\mathbf{q}}}^{k}(\omega)
$$

Once the relaxed interaction forces $\underline{\hat{q}}^{k+1}(\omega)$ are computed, a subsequent step in the iterative procedure is performed until convergence is obtained; an accuracy of $10^{-4}$ is prescribed for the relative residual norms $\left\|\underline{\hat{\mathbf{u}}}_{\mathrm{b}_{2}}^{k+1}(\omega)-\underline{\hat{\mathbf{u}}}_{\mathrm{b}_{2}}^{k}(\omega)\right\| /\left\|\underline{\hat{\mathbf{u}}}_{\mathrm{b}_{2}}^{k+1}(\omega)\right\|$ and $\left\|\underline{\hat{\mathbf{q}}}^{k+1}(\omega)-\underline{\hat{\mathbf{q}}}^{k}(\omega)\right\| /\left\|\underline{\hat{\mathbf{q}}}^{k+1}(\omega)\right\|$ of the interface displacements and interaction forces, respectively.

The choice of a suitable relaxation parameter $\lambda^{k}$ in equation (11) is of great importance in order to ensure and/or speed up the convergence of the iterative algorithm. Constant relaxation parameters are considered, among others, by Elleithy et al. [7, 39] for linear elastostatics, von Estorff et al. [2] for transient elastodynamics, Hagen [40] for fluid-soil-structure interaction, and Grasso [15] for visco-elastodynamics in the frequency domain. Convergence conditions have been established $[7,39]$ and parametric studies have been performed to identify the optimal choice of a constant relaxation parameter [2, 40]. Soares et al. [16] present an iterative procedure for the solution of fluidstructure interaction problems in the frequency domain, in which an optimized relaxation parameter is calculated in each iterative step by minimizing the square error functional of the interface fluxes.

In this paper, Aitken's $\Delta^{2}$-method [17] is employed for the determination of an iteration dependent relaxation parameter $\lambda^{k}$. This method provides a simple but efficient procedure to determine $\lambda^{k}$, based on the results of two subsequent iterations. It is often applied in the iterative 
solution of fluid-structure interaction problems [41, 42] and has already been adopted for transient elastodynamic problems [37]; its application to elastodynamic problems formulated in the frequency domain is not common, however. The methodology is illustrated in figure $2 \mathrm{~b}$ for a general iteration process involving a variable $x$ and a function $f(x)$; the aim is to determine the solution $\bar{x}=f(\bar{x})$ through subsequent evaluations of $f(x)$. An unrelaxed estimation $\tilde{x}^{k+1}=f\left(x^{k}\right)$ is computed in step $k$ of the iterative procedure; if no relaxation is applied (i.e. $\lambda^{k} \equiv 1$ ), the new approximation of $\bar{x}$ yields $x^{k+1}=\tilde{x}^{k+1}$ (cfr. equation (11)). This is illustrated in figure 2a. Application of this procedure would result in a staircase iteration path to the solution. In Aitken's $\Delta^{2}$-method, however, the estimation $\tilde{x}^{k+1}=f\left(x^{k}\right)$ is combined with the result of the previous iteration step $k-1$, which allows for the determination of the new approximation $x^{k+1}$ as the intersection of the linearized function $\tilde{f}^{k}(x)$ through the points $\left\{x^{k-1}, \tilde{x}^{k}=f\left(x^{k-1}\right)\right\}^{\mathrm{T}}$ and $\left\{x^{k}, \tilde{x}^{k+1}=f\left(x^{k}\right)\right\}^{\mathrm{T}}$, and the function $y=x$, respectively. This corresponds to a single step of the secant method [42]. The relaxation parameter $\lambda^{k}$ can hence be written in function of $x^{k-1}, \tilde{x}^{k}, x^{k}$ and $\tilde{x}^{k+1}$ :

$$
\lambda^{k}=\frac{x^{k}-x^{k-1}}{x^{k}-\tilde{x}^{k+1}-x^{k-1}+\tilde{x}^{k}}
$$

while the new approximation yields $x^{k+1}=\lambda^{k} \tilde{x}^{k+1}+\left(1-\lambda^{k}\right) x^{k}$. Introducing the relation $x^{k}-$ $x^{k-1}=\lambda^{k-1}\left(\tilde{x}^{k}-x^{k-1}\right)$ in equation (12) provides a recursive relation for the relaxation parameter $\lambda^{k}$ :

$$
\begin{aligned}
\lambda^{k} & =\lambda^{k-1} \frac{\tilde{x}^{k}-x^{k-1}}{x^{k}-\tilde{x}^{k+1}-x^{k-1}+\tilde{x}^{k}} \\
& =-\lambda^{k-1} \frac{r^{k-1}}{r^{k}-r^{k-1}}
\end{aligned}
$$

with the residual $r^{k}$ defined as $r^{k}=x^{k}-\tilde{x}^{k+1}$.

For the vectorized interaction forces $\hat{\mathbf{q}}(\omega)$ considered in this subsection, however, the division in equation (14) is impossible. Following the approach presented by Irons and Tuck [43], the vectors are projected in the direction $\underline{\hat{r}}^{k}(\omega)-\underline{\hat{\mathbf{r}}}^{k-1}(\omega)=\underline{\hat{\mathbf{q}}}^{k}(\omega)-\underline{\hat{\hat{\mathbf{q}}}}^{k+1}(\omega)-\underline{\hat{\mathbf{q}}}^{k-1}(\omega)+\underline{\hat{\mathbf{q}}}^{k}(\omega)$ :

$$
\lambda^{k}=-\lambda^{k-1} \frac{\left(\underline{\hat{\mathbf{r}}}^{k}(\omega)-\underline{\hat{\hat{\mathbf{r}}}}^{k-1}(\omega)\right)^{\mathrm{T}} \underline{\underline{\hat{\mathbf{r}}}}^{k-1}(\omega)}{\left\|\underline{\hat{\mathbf{r}}}^{k}(\omega)-\underline{\hat{\hat{\mathbf{r}}}}^{k-1}(\omega)\right\|^{2}}
$$

with $\lambda^{0}=1$. Equation (15) can be evaluated at low computational cost, providing a simple and robust way to calculate an iteration dependent relaxation parameter, hence avoiding the need for an empirical trial-and-error process [2]. As all calculations are performed in the frequency domain, the relaxation parameter $\lambda^{k}$ is a complex number. Although the modulus of this number could be limited (e.g. $\left\|\lambda^{k}\right\| \leq 1$ ), this is not done in the present paper, as Soares et al. [16] have observed that faster convergence can be achieved with a non-restricted relaxation parameter for coupled acousticelastodynamic problems in the frequency domain.

An additional reduction of the computation time is achieved by providing an initial guess to the FGMRES solver that is employed to solve equation (5); the relaxed tractions $-\mathbf{T}_{\mathrm{q}}^{-1} \underline{\hat{\mathbf{q}}}^{k}(\omega)$ obtained in iteration $k-1$ are used as an initial guess for the determination of tractions $\underline{\hat{t}}^{k}(\omega)$ in iteration $k$. Using a start vector in the first Neumann-Dirichlet iteration corresponding to the converged solution of the previous frequency step is also advantageous, provided that the frequency bin is sufficiently small.

\subsubsection{Sequential Dirichlet-Neumann algorithm}

The second iterative algorithm considered in this paper consist of a sequential Dirichlet-Neumann scheme; the type of boundary conditions applied to each subdomain is reversed compared to the procedure outlined in subsection 3.2.1. At iteration step $k$, Dirichlet boundary conditions $\underline{\hat{\underline{u}}}_{\mathrm{b}_{2}}^{k}(\omega)$ are imposed on the interface $\Sigma$ of the FE subdomain, providing the interaction forces $\underline{\hat{q}}^{k}(\omega)$ through 

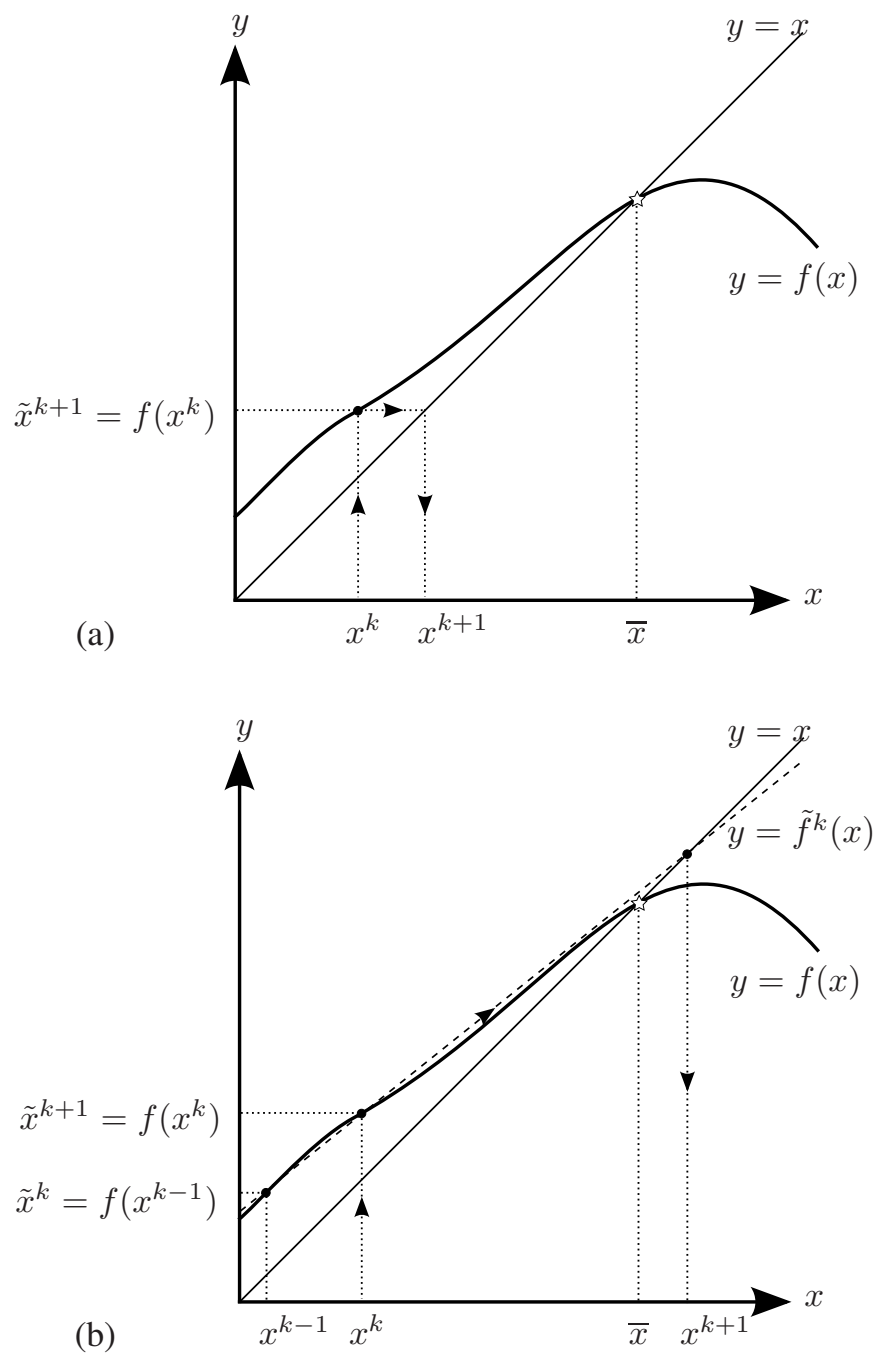

Figure 2. Interface relaxation technique employed in sequential iterative algorithms, involving the functions $y=x$ (thin solid line), $y=f(x)$ (thick solid line) and $y=\tilde{f}^{k}(x)$ (dashed line). The target solution $\bar{x}=f(\bar{x})$ is indicated with a star. The procedure (a) without relaxation is compared to (b) Aitken's $\Delta^{2}$-method.

the solution of equation (9) (and condensation of the internal degrees of freedom $\underline{\hat{\underline{u}}}_{\mathrm{b}_{1}}^{k}(\omega)$ ). The corresponding interface tractions $\underline{\hat{\mathbf{t}}}^{k}(\omega)=-\mathbf{T}_{\mathrm{q}}^{-1} \underline{\hat{\mathbf{q}}}^{k}(\omega)$ are applied to the BE subdomain, and unrelaxed interface displacements $\underline{\hat{\mathrm{u}}}_{\mathrm{b}_{2}}^{k+1}(\omega)$ are obtained by solving the preconditioned system of equations (5) using the FGMRES solver. Aitken's $\Delta^{2}$-method is finally employed for the determination of an optimized relaxation parameter $\lambda^{k}$ (with equation (15) now based on interface displacements instead of interaction forces), allowing for the computation of relaxed interface displacements $\underline{\hat{\mathbf{u}}}_{\mathrm{b}_{2}}^{k+1}(\omega)$. This iterative procedure is repeated until convergence is achieved.

\subsubsection{Parallel Neumann-Neumann algorithm}

A parallel Neumann-Neumann iterative scheme is obtained if the interaction forces $\underline{\hat{q}}^{k}(\omega)$ and corresponding interface tractions $\hat{\mathbf{t}}^{k}(\omega)=-\mathbf{T}_{\mathbf{q}}^{-1} \underline{\hat{\mathbf{q}}}^{k}(\omega)$ are simultaneously imposed as Neumann boundary conditions on the interface $\Sigma$ of the FE and BE subdomain, respectively. Solving equations (9) and (5) provides (incompatible) interface displacements $\underline{\hat{\mathbf{u}}}_{\mathrm{b}_{2}, \mathrm{FE}}^{k}(\omega)$ and $\underline{\hat{\underline{\hat{u}}}}_{\mathrm{b}_{2}, \mathrm{BE}}^{k}(\omega)$, respectively. The discrepancy of interface displacements $\Delta \underline{\hat{\mathbf{u}}}_{2}^{k}(\omega)=\underline{\hat{\mathbf{u}}}_{\mathrm{b}_{2}, \mathrm{BE}}^{k}(\omega)-\underline{\hat{\mathbf{u}}}_{\mathrm{b}_{2}, \mathrm{FE}}^{k}(\omega)$ is subsequently 
employed to calculate an increment of interaction forces $\Delta \underline{\hat{q}}^{k}(\omega)$. The displacement discrepancy $\Delta \underline{\hat{\underline{u}}}_{\mathrm{b}_{2}}^{k}(\omega)$ can either be imposed on the FE or the BE subdomain to compute this increment; this either yields unrelaxed interaction forces $\underline{\hat{\mathbf{q}}}_{\mathrm{FE}}^{k+1}(\omega)=\underline{\hat{\mathbf{q}}}^{k}(\omega)+\Delta \underline{\hat{\mathbf{q}}}_{\mathrm{FE}}^{k}(\omega)$ or $\underline{\hat{\mathbf{q}}}_{\mathrm{BE}}^{k+1}(\omega)=\underline{\hat{\mathbf{q}}}^{k}(\omega)+$ $\Delta \underline{\hat{q}}_{\mathrm{BE}}^{k}(\omega)$. Application of Aitken relaxation finally provides relaxed interaction forces $\underline{\hat{q}}_{\mathrm{FE}}^{k+1}(\omega)$ or $\underline{\hat{\mathbf{q}}}_{\mathrm{BE}}^{k+1}(\omega)$, which are used in a subsequent step of the iterative scheme.

One can expect that the fastest convergence will be achieved if the force increment $\Delta \underline{\hat{q}}^{k}(\omega)$ is calculated by imposing the displacement discrepancy $\Delta \underline{\underline{\hat{u}}}_{\mathrm{b}_{2}}^{k}(\omega)$ on the most flexible subdomain, as this will yield the smallest increment $\Delta \hat{\mathbf{q}}^{k}(\omega)$. It is difficult, however, to quantify the flexibility of each subdomain a priori, especially as the latter is frequency dependent. Choosing either the FE or the BE subdomain for the calculation of $\Delta \hat{\mathbf{q}}^{k}(\omega)$ hence requires an understanding of the dynamic behaviour of each subdomain; a wrong choice can significantly deteriorate the convergence of the iterative procedure.

A novel and more robust variant of the algorithm is therefore presented in this paper, where $\underline{\hat{\mathbf{q}}}_{\mathrm{FE}}^{k+1}(\omega)$ and $\underline{\hat{\mathbf{q}}}_{\mathrm{BE}}^{k+1}(\omega)$ are simultaneously accounted for in the determination of the relaxed interaction forces $\hat{\mathbf{q}}^{k+1}(\omega)$. The concept is illustrated in figure 3 for a general iteration process in terms of a variable $x$ and two functions $f(x)$ and $g(x)$, with solution $\bar{x}=f(\bar{x})=g(\bar{x})$. The proposed approach is based on a simultaneous application of Aitken relaxation to $f(x)$ and $g(x)$. At iteration step $k$, two estimations $\tilde{x}_{f}^{k+1}=f\left(x^{k}\right)$ and $\tilde{x}_{g}^{k+1}=g\left(x^{k}\right)$ are calculated. These estimations are combined with the data points $\left\{x^{k-1}, \tilde{x}_{f}^{k}=f\left(x^{k-1}\right)\right\}^{\mathrm{T}}$ and $\left\{x^{k-1}, \tilde{x}_{g}^{k}=g\left(x^{k-1}\right)\right\}^{\mathrm{T}}$ obtained in the previous iteration step $k-1$ to define linear approximations $\tilde{f}^{k}(x)$ and $\tilde{g}^{k}(x)$ of the functions $f(x)$ and $g(x)$, respectively. The ordinate of the intersection of these linearized functions $\tilde{f}^{k}(x)$ and $\tilde{g}^{k}(x)$ provides a new approximation $x^{k+1}$ of the solution $\bar{x}$ :

$$
x^{k+1}=\frac{r_{g}^{k}}{r_{g}^{k}-r_{f}^{k}} \tilde{x}_{f}^{k+1}-\frac{r_{f}^{k}}{r_{g}^{k}-r_{f}^{k}} \tilde{x}_{g}^{k+1}
$$

with $r_{f}^{k}=\tilde{x}_{f}^{k+1}-\tilde{x}_{f}^{k}$ and $r_{g}^{k}=\tilde{x}_{g}^{k+1}-\tilde{x}_{g}^{k}$. These residual vectors are defined differently compared to equation (14).

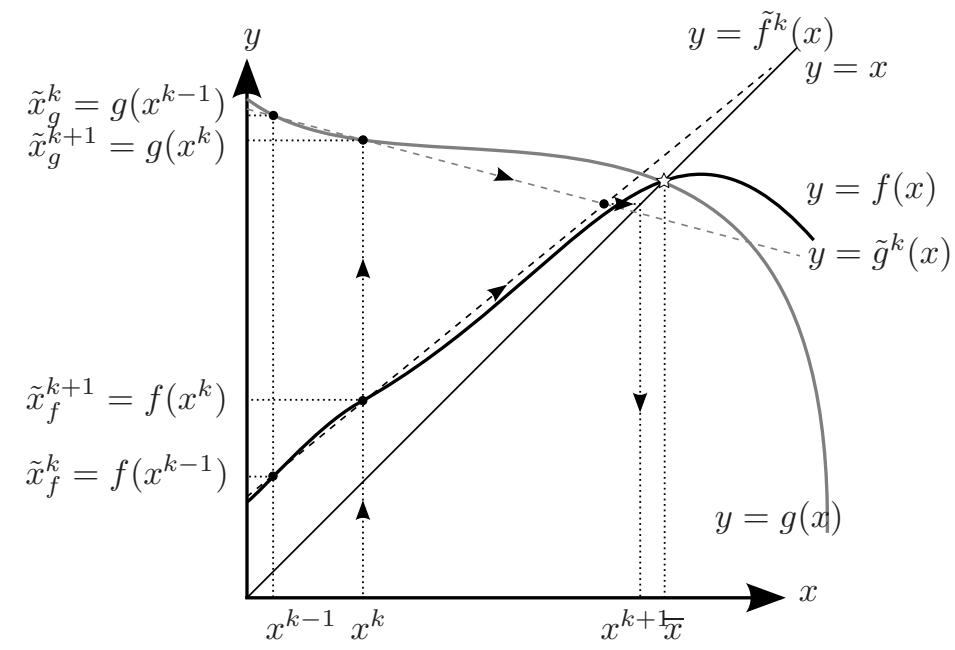

Figure 3. Interface relaxation technique employed in parallel iterative algorithms, involving the functions $y=f(x)$ (black solid line), $y=g(x)$ (grey solid line), $y=\tilde{f}^{k}(x)$ (black dashed line), $y=\tilde{g}^{k}(x)$ (grey dashed line), and $y=x$ (thin solid line). The target solution $\bar{x}=f(\bar{x})=g(\bar{x})$ is indicated with a star. 
For the vectorized interaction forces $\underline{\hat{\mathbf{q}}}(\omega)$, a projection in the direction $\underline{\hat{\mathbf{r}}}_{\mathrm{BE}}^{k}(\omega)-\underline{\hat{\hat{r}}}_{\mathrm{FE}}^{k}(\omega)=$ $\underline{\hat{\mathbf{q}}}_{\mathrm{BE}}^{k+1}(\omega)-\underline{\hat{\mathbf{q}}}_{\mathrm{BE}}^{k}(\omega)-\underline{\hat{\mathbf{q}}}_{\mathrm{FE}}^{k+1}(\omega)+\underline{\hat{\mathbf{q}}}_{\mathrm{FE}}^{k}(\omega)$ is introduced in equation (16):

$$
\underline{\hat{\mathbf{q}}}^{k+1}(\omega)=\frac{\left(\underline{\hat{\mathbf{r}}}_{\mathrm{BE}}^{k}(\omega)-\underline{\hat{\mathbf{r}}}_{\mathrm{FE}}^{k}(\omega)\right)^{\mathrm{T}} \underline{\underline{\hat{\mathbf{r}}}}_{\mathrm{BE}}^{k}(\omega)}{\left\|\underline{\hat{\mathbf{r}}}_{\mathrm{BE}}^{k}(\omega)-\underline{\hat{\mathbf{r}}}_{\mathrm{FE}}^{k}(\omega)\right\|^{2}} \underline{\hat{\mathbf{q}}}_{\mathrm{FE}}^{k+1}(\omega)-\frac{\left(\underline{\hat{\mathbf{r}}}_{\mathrm{BE}}^{k}(\omega)-\underline{\hat{\mathbf{r}}}_{\mathrm{FE}}^{k}(\omega)\right)^{\mathrm{T}} \underline{\underline{\mathbf{r}}}_{\mathrm{FE}}^{k}(\omega)}{\left\|\underline{\hat{\mathbf{r}}}_{\mathrm{BE}}^{k}(\omega)-\underline{\hat{\mathbf{r}}}_{\mathrm{FE}}^{k}(\omega)\right\|^{2}} \underline{\hat{\mathbf{q}}}_{\mathrm{BE}}^{k+1}(\omega)
$$

Equation (17) clearly indicates that $\underline{\hat{\mathbf{q}}}_{\mathrm{FE}}^{k+1}(\omega)$ and $\underline{\hat{\mathrm{q}}}_{\mathrm{BE}}^{k+1}(\omega)$ are simultaneously accounted for in the determination of a new estimate $\hat{\mathbf{q}}^{k+1}(\omega)$, with iteration dependent weighting factors based on data of two subsequent iterations; these weighting factors can be calculated at low computational cost. This approach hence avoids the need for a priori information concerning the flexibility of the FE and BE subdomain.

\subsubsection{Parallel Dirichlet-Dirichlet algorithm}

The final iterative algorithm discussed in this paper is a parallel Dirichlet-Dirichlet strategy, which is very similar to the procedure outlined in subsection 3.2.3. Imposing Dirichlet boundary conditions $\underline{\hat{\mathbf{u}}}_{\mathrm{b}_{2}}^{k}(\omega)$ on $\Sigma$ allows for the computation of interaction forces $\underline{\hat{\mathbf{q}}}_{\mathrm{FE}}^{k}(\omega)$ and $\underline{\hat{\mathbf{q}}}_{\mathrm{BE}}^{k}(\omega)$ through equations (9) and (5), respectively. The resulting force discrepancy $\Delta \underline{\hat{\mathbf{q}}}^{\bar{k}}(\omega)=\underline{\hat{\mathbf{q}}}_{\mathrm{BE}}^{k}(\omega)-\underline{\hat{\mathbf{q}}}_{\mathrm{FE}}^{k}(\omega)$ is employed to calculate interface displacement increments $\Delta \underline{\hat{\mathbf{u}}}_{\mathrm{b}_{2}, \mathrm{FE}}^{k}(\omega)$ and $\Delta \underline{\hat{\mathbf{u}}}_{\mathrm{b}_{2}, \mathrm{BE}}^{k}(\omega)$, and unrelaxed displacements $\underline{\hat{\hat{\mathbf{u}}}}_{\mathrm{b}_{2}, \mathrm{FE}}^{k+1}(\omega)=\underline{\hat{\mathbf{u}}}_{\mathrm{b}_{2}, \mathrm{FE}}^{k}(\omega)+\Delta \underline{\hat{\mathbf{u}}}_{\mathrm{b}_{2}, \mathrm{FE}}^{k}(\omega)$ and $\underline{\hat{\mathbf{u}}}_{\mathrm{b}_{2}, \mathrm{BE}}^{k+1}(\omega)=\underline{\hat{\mathbf{u}}}_{\mathrm{b}_{2}, \mathrm{BE}}^{k}(\omega)+\Delta \underline{\hat{\mathbf{u}}}_{\mathrm{b}_{2}, \mathrm{BE}}^{k}(\omega)$ can subsequently be obtained. The relaxed interface displacements $\underline{\hat{\mathbf{u}}}_{\mathrm{b}_{2}}^{k+1}(\omega)$ are finally computed by means of the relaxation procedure introduced in subsection 3.2.3; equation (17) in that case is based on interface displacements instead of interaction forces. The procedure is repeated until convergence is achieved.

\subsection{Monolithic FE- $\mathscr{H}-B E$ coupling}

The coupling of FE and $\mathscr{H}$-BE models can also performed by means of a monolithic approach, in which the governing equations of both subdomains are solved simultaneously, while the assembly of a dynamic soil stiffness matrix is avoided. This approach fundamentally differs from the conventional direct coupling approach outlined in subsection 3.1 (equation (6)). Combining equations (1) and (3) and accounting for continuity of displacements and equilibrium of tractions at the soil-structure interface $\Sigma$ yields:

$$
\left[\begin{array}{ccc}
\widehat{\mathbf{K}}_{\mathrm{b}_{1} \mathrm{~b}_{1}}(\omega) & \widehat{\mathbf{K}}_{\mathrm{b}_{1} \mathrm{~b}_{2}}(\omega) & \mathbf{0} \\
\widehat{\mathbf{K}}_{\mathrm{b}_{2} \mathrm{~b}_{1}}(\omega) & \widehat{\mathbf{K}}_{\mathrm{b}_{2} \mathrm{~b}_{2}}(\omega) & \mathbf{T}_{\mathrm{q}} \\
\mathbf{0} & \widehat{\mathbf{T}}_{\mathscr{H}}(\omega)+\mathbf{I} & -\widehat{\mathbf{U}}_{\mathscr{H}}(\omega)
\end{array}\right]\left\{\begin{array}{c}
\hat{\underline{\mathbf{u}}}_{\mathrm{b}_{1}}(\omega) \\
\underline{\hat{\mathbf{u}}}_{\mathrm{b}_{2}}(\omega) \\
\underline{\hat{\mathbf{t}}}(\omega)
\end{array}\right\}=\left\{\begin{array}{c}
\hat{\hat{\mathbf{f}}}_{\mathrm{b}_{1}}(\omega) \\
\underline{\mathbf{f}}_{\mathrm{b}_{2}}(\omega) \\
\underline{\mathbf{0}}
\end{array}\right\}+\left\{\begin{array}{c}
\underline{\mathbf{0}} \\
\hat{\hat{\mathbf{f}}}_{\mathrm{b}_{2}}^{\mathrm{s}}(\omega) \\
\underline{\mathbf{0}}
\end{array}\right\}
$$

where the coupling matrix $\mathbf{T}_{\mathrm{q}}$ is defined in equation (7). The system size in this monolithic approach is $\left(\left(n_{\text {dof }_{1}}+2 n_{\text {dof }_{2}}\right) \times\left(n_{\text {dof }_{1}}+2 n_{\text {dof }_{2}}\right)\right)$, which is significantly larger than in the classical direct coupling strategy of subsection 3.1. The coefficient matrix is never assembled explicitly, however, as equation (18) is solved by means of an iterative GMRES solver. This requires an efficient evaluation of the matrix-vector product, indicating that the monolithic formulation (18) is only advantageous if a fast $\mathrm{BE}$ method (in casu a formulation based on $\mathscr{H}$-matrices) is employed. A monolithic approach is rarely used in elastodynamics [15]; the monolithic coupling of FE and fast multipole BE models presented by Margonari et al. [8] remains restricted to elastostatics. This strategy is more often applied for solving strongly coupled fluid-structure interaction problems [18, 19], as discretization methods commonly used for the fluid and the structure lead to sparse matrices.

The coefficient matrix in equation (18) is likely to be ill-conditioned, as the matrix entries arising from the $\mathrm{FE}$ and $\mathrm{BE}$ discretizations differ by several orders of magnitude. Convergence of the iterative solver will therefore be slow, and the incorporation of a suitable preconditioner is 
indispensable. The following simple right preconditioner $\widehat{\mathbf{M}}(\omega)$ is employed:

$$
\widehat{\mathbf{M}}(\omega)=\left[\begin{array}{ccc}
\operatorname{diag}\left(\widehat{\mathbf{K}}_{\mathrm{b}_{1} \mathrm{~b}_{1}}(\omega)\right) & \mathbf{0} & \mathbf{0} \\
\mathbf{0} & \operatorname{diag}\left(\widehat{\mathbf{K}}_{\mathrm{b}_{2} \mathrm{~b}_{2}}(\omega)\right) & \mathbf{T}_{\mathrm{q}} \\
\mathbf{0} & \operatorname{blkdiag}\left(\widehat{\mathbf{T}}_{\mathscr{H}}(\omega)\right)+\mathbf{I} & \operatorname{blkdiag}\left(-\widehat{\mathbf{U}}_{\mathscr{H}}(\omega)\right)
\end{array}\right]
$$

where the size of the diagonal blocks of $\widehat{\mathbf{T}}_{\mathscr{H}}(\omega)$ and $\widehat{\mathbf{U}}_{\mathscr{H}}(\omega)$ is determined by the lowest hierarchical BE cluster level. The nested FGMRES solution procedure discussed in subsection 2.2 is employed here as well, avoiding the explicit assembly of $\widehat{\mathbf{M}}^{-1}(\omega)$.

\section{VERIFICATION EXAMPLES}

In the following subsections, two examples are considered to verify the numerical implementation of the proposed $\mathrm{FE}-\mathscr{H}$-BE coupling algorithms and to assess their computational performance. While the first example is related to a full space geometry, the second example involves Green's functions for a layered halfspace. All calculations have been performed on Intel ${ }^{\circledR}$ Xeon $^{\circledR}$ E5520 (2.26 GHz) CPUs.

\subsection{D spherical cavity embedded in a layered space}

Consider a 3D spherical cavity with inner radius $r_{\mathrm{i}}=1 \mathrm{~m}$ embedded in a layered space, consisting of a spherical layer with outer radius $r_{\mathrm{o}}=2 \mathrm{~m}$ and a homogeneous full space (figure $4 \mathrm{a}$ ). The cavity is loaded by an internal pressure $\hat{p}(\omega)=1 \mathrm{~Pa} / \mathrm{Hz}$. The full space is characterized by a dilatational wave velocity $C_{\mathrm{p}}=300 \mathrm{~m} / \mathrm{s}$, a density $\rho=1800 \mathrm{~kg} / \mathrm{m}^{3}$, and a material damping ratio $\beta_{\mathrm{p}}=0.025$ in volumetric deformation. The dilatational wave velocity in the spherical layer is defined as $\alpha C_{\mathrm{p}}$, where the following values are considered for the ratio $\alpha$ : (i) $\alpha=1 / 2$, (ii) $\alpha=1$ and (iii) $\alpha=2$. The same material damping ratio and density as in the full space are used.

(a)

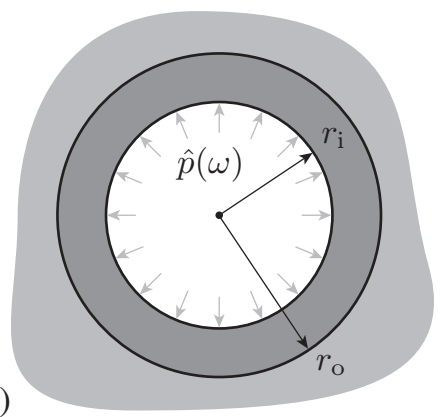

Figure 4. (a) 3D spherical cavity with inner radius $r_{\mathrm{i}}$ embedded in a layered space, consisting of spherical layer with outer radius $r_{\mathrm{o}}=2 \mathrm{~m}$ and a homogeneous full space, and subjected to an internal pressure $\hat{p}(\omega)$. Half of the FE discretization of the spherical layer is shown in (b).

The spherical layer is discretized by means of 6000 eight-node solid finite elements, which are coupled to a conforming BE mesh consisting of 600 four-node quadrilateral elements on the soilstructure interface (figure $4 \mathrm{~b}$ ). A nodal collocation scheme is used for the latter to facilitate the FE$\mathrm{BE}$ coupling, resulting in $19866 \mathrm{FE}$ and $1806 \mathrm{BE}$ degrees of freedom. This results in 6.67 elements per dilatational wavelength $\lambda_{\mathrm{p}}=\alpha C_{\mathrm{p}} / f$ at a frequency of $100 \mathrm{~Hz}$ for $\alpha=1 / 2$. Analytical full space fundamental solutions [14] are employed in the $\mathscr{H}$-BE formulation.

Each of the FE- $\mathscr{H}$-BE coupling strategies outlined in the section 3 is employed to calculate the response in the frequency range between $0 \mathrm{~Hz}$ and $100 \mathrm{~Hz}$, with a frequency step of $1 \mathrm{~Hz}$. A maximum of 200 iterations is prescribed for the iterative coupling algorithms. The sequential Neumann-Dirichlet algorithm presented in subsection 3.2.1 is unable to determine the static 
solution, as application of Neumann boundary conditions to the unconstrained structural domain $\Omega_{\mathrm{b}}$ results in singular FE equations. The convergence behaviour at low frequencies is hence expected to be poor; the frequency sweep is therefore performed from high to low frequencies. The sequential Dirichlet-Neumann algorithm of subsection 3.2.2, on the other hand, succeeds in solving the coupled problem for static excitation, as displacements are imposed on the boundary of the FE subdomain.

Figure 5 shows the real and imaginary part of the radial displacement at $r=r_{\mathrm{o}}$, for the three values of the wave velocity ratio $\alpha$. All methods yield accurate results in very good agreement with the analytical solution [44] for all values of the ratio $\alpha$, with exception of the sequential NeumannDirichlet algorithm, which is unable to retrieve the correct solution within the prescribed number of iterations at $[12,31-38,41-42,46,49-62,64-80,82-100] \mathrm{Hz}$ for a wave velocity ratio $\alpha=1 / 2$. At these particular frequencies, the relative residual norm of the interface displacements and interaction forces still exceeds the specified accuracy of $10^{-4}$.

(a)
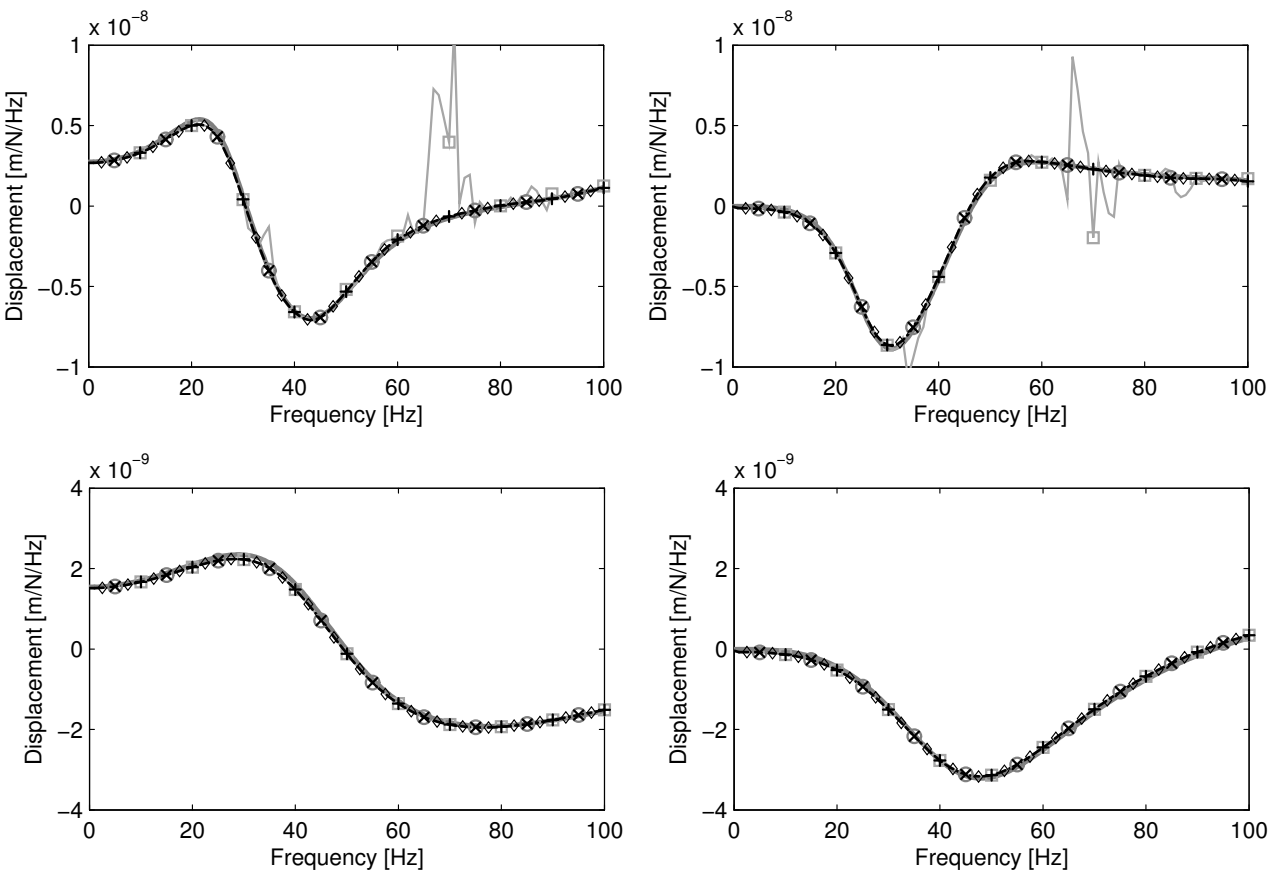

(b)


Figure 5. Real (left) and imaginary (right) part of the radial displacement at $r=r_{\mathrm{o}}$ due to a unit harmonic pressure applied to a spherical cavity embedded in a layered space, for (a) $\alpha=1 / 2$, (b) $\alpha=1$ and (c) $\alpha=2$. The solutions of the classical direct coupling approach (dashed black line), the iterative Neumann-Dirichlet (grey squares), Dirichlet-Neumann (black plus signs), Neumann-Neumann (grey circles) and DirichletDirichlet (black crosses) algorithms, and the monolithic coupling procedure (black rhombuses) are compared to the analytical solution (solid grey line) [44]. The markers are only drawn at a limited number of data points. 
The integral representation theorem subsequently allows for the computation of the radiated wavefield in the full space from the displacements and tractions on the FE- $\mathscr{H}$-BE interface. Figure 6 shows the real and imaginary part of the radial displacement at $r=10 \mathrm{~m}$. The solutions of the various coupling procedures are clearly in good correspondence with each other and agree with the analytical solution, except for the Neumann-Dirichlet algorithm at the aforementioned frequencies for the case $\alpha=1 / 2$.

(a)
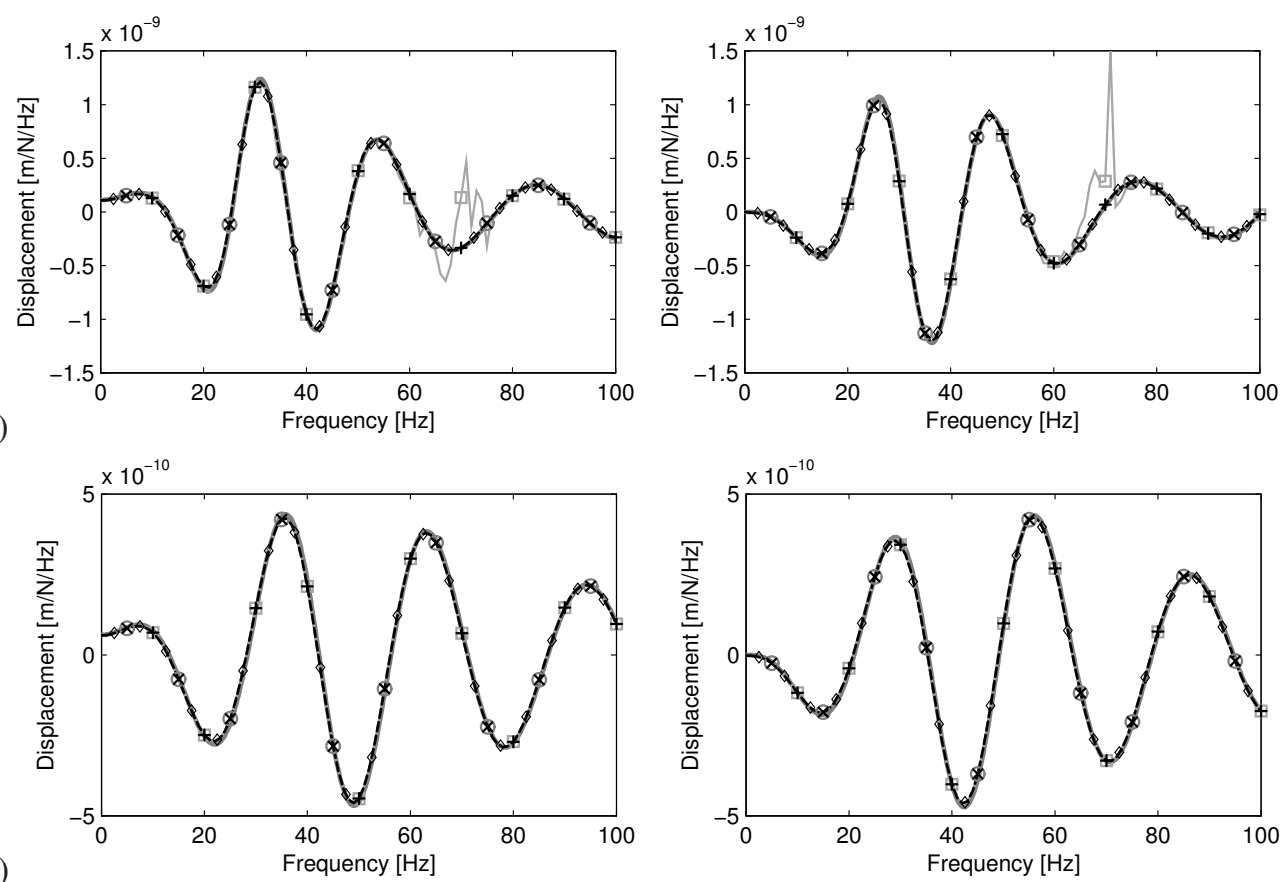

(b)
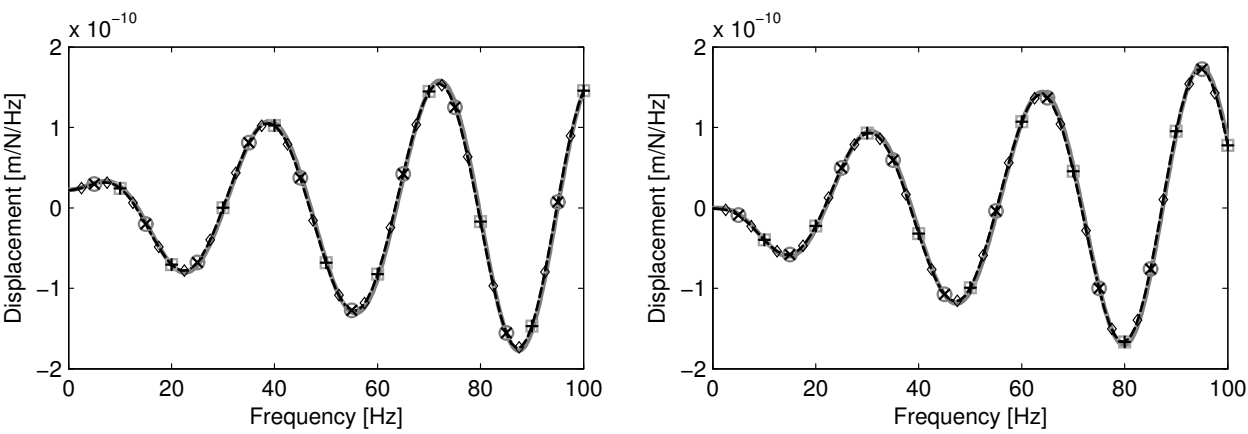

Figure 6. Real (left) and imaginary (right) part of the radial displacement at $r=10 \mathrm{~m}$ due to a unit harmonic pressure applied to a spherical cavity embedded in a layered space, for (a) $\alpha=1 / 2$, (b) $\alpha=1$ and (c) $\alpha=2$. The solutions of the classical direct coupling approach (dashed black line), the iterative Neumann-Dirichlet (grey squares), Dirichlet-Neumann (black plus signs), Neumann-Neumann (grey circles) and DirichletDirichlet (black crosses) algorithms, and the monolithic coupling procedure (black rhombuses) are compared to the analytical solution (solid grey line) [44]. The markers are only drawn at a limited number of data points.

Figures 5 and 6 validate the numerical implementation of the $\mathrm{FE}-\mathscr{H}$-BE coupling strategies presented in this paper. The computational performance of each method strongly differs, however. Figure 7 shows the CPU time required in each algorithm as a function of the frequency, for the three wave velocity ratios considered. It is observed that the computation time in the direct coupling approach significantly exceeds the computational effort of the alternative procedures due to the drawbacks summarized in subsection 3.1, rendering the conventional method the least efficient. 
The computation time remains quasi independent of the wave velocity ratio, but increases with frequency. This is caused by an increase of the time required for the assembly and solution of the $\mathscr{H}$-BE equations at higher frequencies [12]; a similar trend is also observed for the other coupling methodologies. The computational efficiency of the iterative coupling schemes, on the other hand, shows a much stronger correlation with the wave velocity ratio $\alpha$. This is in particular the case for the sequential variants: the CPU time in the Neumann-Dirichlet algorithm strongly decreases for increasing values of $\alpha$, while the reverse is observed for the Dirichlet-Neumann approach. This indicates that Neumann boundary conditions should be applied to the most stiff subdomain in order to achieve the fastest convergence. The efficiency of the parallel iterative algorithms depends less strongly on $\alpha$ due to the novel relaxation procedure introduced in subsection 3.2.3, as the contribution of each subdomain to the relaxed interaction forces or displacements is balanced in every step of the iterative procedure. Finally, figure 7 illustrates that the monolithic coupling scheme is also relatively insensitive to the value of $\alpha$, but the overall computational performance of this methodology remains relatively poor compared to the iterative algorithms. The implementation of a more rigorous preconditioner than the one applied in this paper might result in an improved convergence behaviour. Algebraic multigrid preconditioning strategies adapted for hierarchical matrices [45] or approaches based on the sparse approximate inverse (SPAI) of the BE matrices [8] are worthwhile to be considered in future research.

Some important conclusions can be drawn from the results presented in figure 7. It is clear that the classical direct approach is not well suited to provide an efficient coupling of FE and $\mathscr{H}$-BE models. In particular, if there exists a strong stiffness contrast between the subdomains, a sequential iterative algorithm is preferred. It is recommended to impose Neumann boundary conditions on the most stiff subdomain; the reverse choice can significantly deteriorate the convergence behaviour. If such a contrast is not apparent, however, the parallel iterative algorithms as well as the monolithic approach provide a reliable and robust coupling of FE and $\mathscr{H}$-BE models.

The efficiency of the sequential iterative procedures is now investigated in more detail. In each global iterative step $k$, an iterative FGMRES solver is used to solve equation (5) for tractions $\underline{\hat{\mathbf{t}}}^{k}(\omega)$ or interface displacements $\underline{\hat{\mathbf{u}}}_{\mathrm{b}_{2}}^{k+1}(\omega)$ in the Neumann-Dirichlet or Dirichlet-Neumann algorithm, respectively. Figures 8 and 9 show the number of FGMRES-iterations for the solution of equation (5) as a function of the frequency and the iteration step $k$. The number of FGMRESiterations generally decreases in subsequent iteration steps, as the relaxed solution obtained in iteration $k-1$ is used as an initial guess in the FGMRES solver in iteration $k$. The number of global iterations strongly decreases for increasing values of the wave velocity ratio $\alpha$ in the NeumannDirichlet algorithm (figure 8), while the reverse is observed in the Dirichlet-Neumann approach (figure 9).

Numerical attempts have furthermore demonstrated that application of Aitken's $\Delta^{2}$-method for the interface relaxation is crucial in order to ensure convergence in the sequential iterative algorithms. No convergence could be obtained with these algorithms in any of the examples in case a fixed value was attributed to the relaxation parameter $\lambda^{k}$ (several constant values between 0 and 1 have been considered), in the entire frequency range of interest. Figure 10 shows the fluctuation of $\lambda^{k}$ in the sequential Neumann-Dirichlet algorithm at a frequency of $100 \mathrm{~Hz}$ for $\alpha=1$, clearly illustrating how the relaxation parameter changes throughout the iteration steps.

Finally, the effectiveness of the novel relaxation procedure for the parallel iterative algorithms introduced in subsection 3.2.3 is demonstrated. Figure 11 compares the CPU time in the iterative Neumann-Neumann algorithm using equation (17) for the determination of relaxed interaction forces $\underline{\hat{q}}^{k+1}(\omega)$ to the cases where either $\underline{\hat{q}}_{\mathrm{FE}}^{k+1}(\omega)$ or $\underline{\hat{q}}_{\mathrm{BE}}^{k+1}(\omega)$ is employed, respectively. As indicated in subsection 3.2.3, the fastest convergence is achieved if the displacement discrepancy $\Delta \underline{\hat{\underline{u}}}_{\mathrm{b}_{2}}^{k}(\omega)$ is imposed on the most flexible subdomain, i.e. on the FE subdomain for $\alpha=1 / 2$ and on the BE subdomain for $\alpha=2$. The reverse choice strongly affects the convergence behaviour and the algorithm does even not convergence at some frequencies if $\underline{\hat{q}}_{\mathrm{BE}}^{k+1}(\omega)$ is employed for $\alpha=1 / 2$. The relaxation procedure corresponding to equation (17) proves to be a reliable alternative, as the associated CPU times only moderately depend on $\alpha$ and are often bounded by the computation times of the two other approaches. 


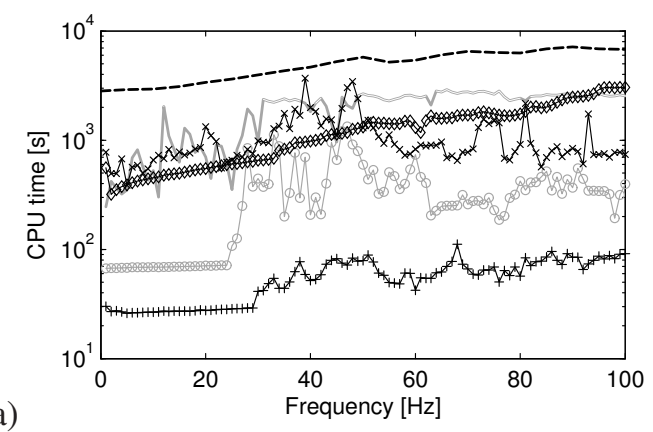

(a)

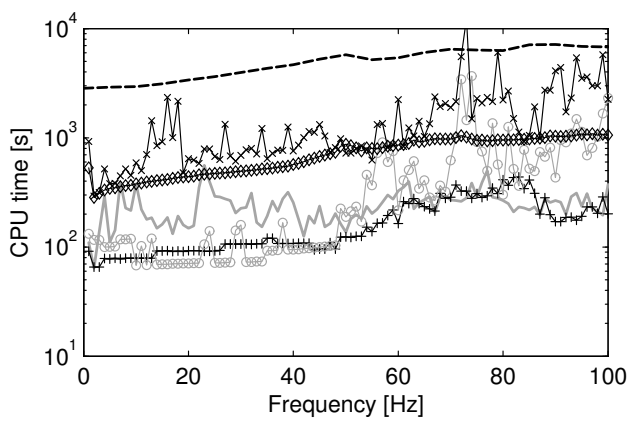

(b)

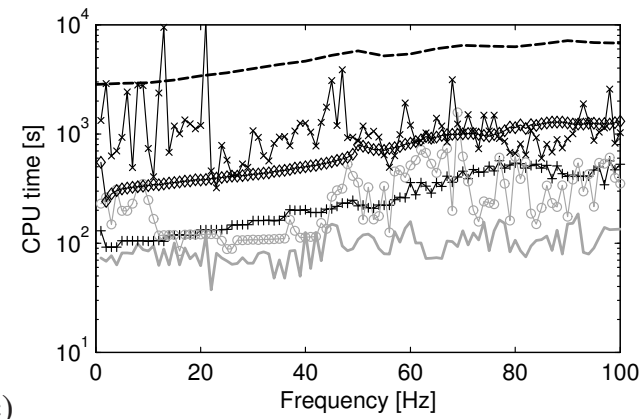

Figure 7. CPU time required in the classical direct coupling approach (dashed black line), the iterative Neumann-Dirichlet (solid grey line), Dirichlet-Neumann (black plus signs), Neumann-Neumann (grey circles) and Dirichlet-Dirichlet (black crosses) algorithms, and the monolithic coupling procedure (black rhombuses), for a spherical cavity embedded in a layered space with (a) $\alpha=1 / 2$, (b) $\alpha=1$ and (c) $\alpha=2$.

The lines are only drawn if convergence is achieved.

\subsection{Flexible surface foundation on a horizontally layered halfspace}

A flexible square surface foundation on a horizontally layered halfspace is considered in this subsection. The concrete foundation has dimensions $5 \mathrm{~m} \times 5 \mathrm{~m} \times 0.25 \mathrm{~m}$ and a Young's modulus $E=33 \mathrm{GPa}$, a Poisson's ratio $\nu=0.20$, and a density $\rho=2500 \mathrm{~kg} / \mathrm{m}^{3}$. Rayleigh damping with a modal damping ratio $\xi=0.03$ in the first two flexible modes is used. A unit harmonic vertical point excitation is applied at the center of the foundation, within a frequency range between $0 \mathrm{~Hz}$ and $100 \mathrm{~Hz}$.

The soil consists of two layers on a halfspace, each with a thickness of $2 \mathrm{~m}$. The shear wave velocity $C_{\mathrm{s}}$ is equal to $150 \mathrm{~m} / \mathrm{s}$ in the top layer, $250 \mathrm{~m} / \mathrm{s}$ in the second layer, and $300 \mathrm{~m} / \mathrm{s}$ in the underlying halfspace. The Poisson's ratio $\nu$ is $1 / 3$ everywhere, resulting in dilatational wave velocities $C_{\mathrm{p}}$ of $300 \mathrm{~m} / \mathrm{s}, 500 \mathrm{~m} / \mathrm{s}$, and $600 \mathrm{~m} / \mathrm{s}$, respectively. Material damping ratios $\beta_{\mathrm{s}}=\beta_{\mathrm{p}}=$ 0.025 in both deviatoric and volumetric deformation are attributed to the layers and the halfspace, while a uniform density $\rho=1800 \mathrm{~kg} / \mathrm{m}^{3}$ is considered throughout the medium. 


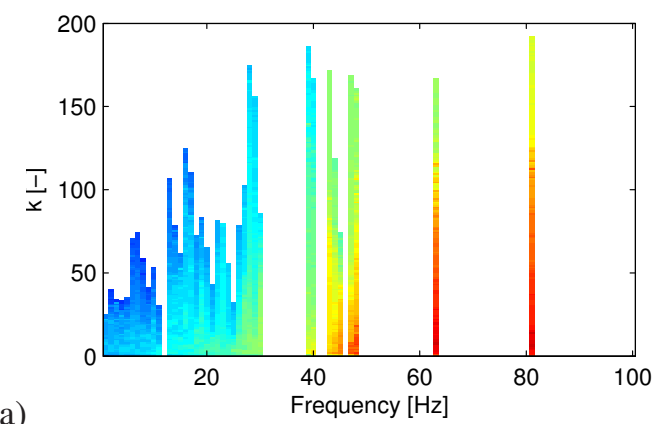

(a)

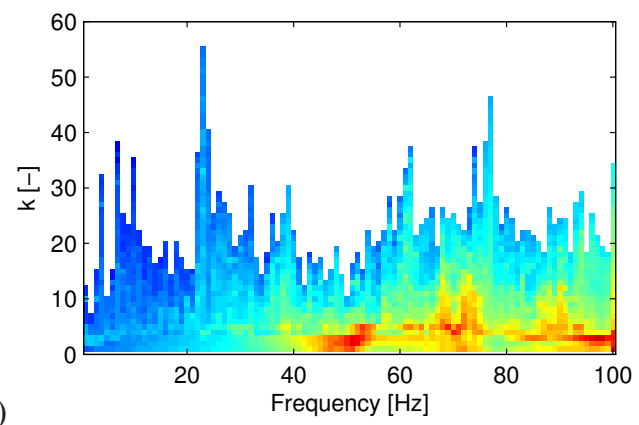

(b)

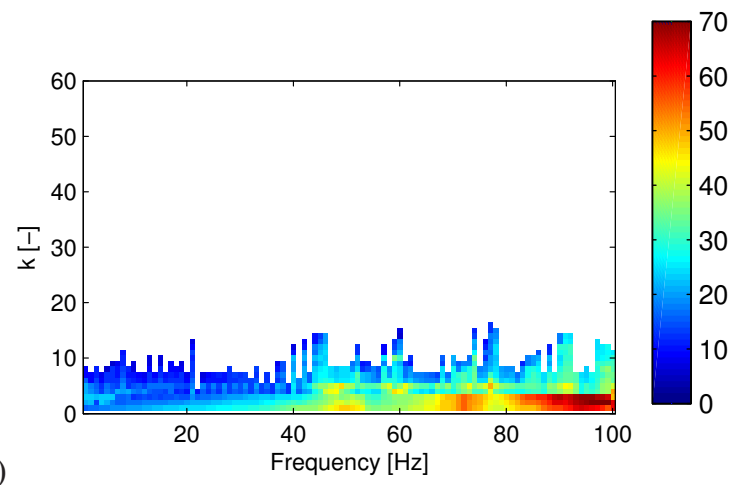

Figure 8. Number of FGMRES-iterations (colour code) to solve the $\mathscr{H}$-BE equation (5) for tractions in the iterative Neumann-Dirichlet algorithm as a function of the frequency and the iteration step $k$, for a spherical cavity embedded in a layered space with (a) $\alpha=1 / 2$, (b) $\alpha=1$ and (c) $\alpha=2$. The bars are only drawn if convergence is achieved.

The foundation is discretized by means of $30 \times 30$ square Kirchhoff plate elements, which are coupled to a conforming BE mesh for the soil using a nodal collocation scheme. Up to nine elements per shear wavelength $\lambda_{\mathrm{s}}=C_{\mathrm{s}} / f$ are provided at the maximum frequency of $100 \mathrm{~Hz}$ (determined by the shear wave velocity of the top layer). Green's functions for a layered halfspace are incorporated in the $\mathscr{H}$-BE formulation $[12,13,46]$, avoiding the necessity to discretize the free surface and the layer interfaces. It is expected that the foundation will behave much stiffer than the soil in the frequency range of interest. In accordance with the findings of subsection 4.1, an iterative NeumannDirichlet coupling scheme is therefore employed. No analytical reference solution is available for this problem, however, and the monolithic coupling approach is therefore considered as well to provide verification. 
(a)
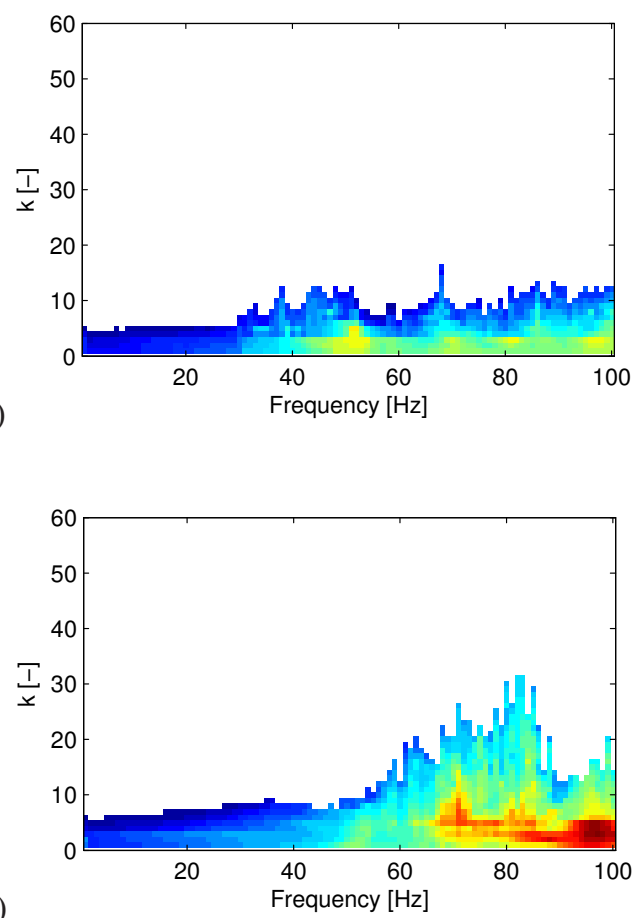

(b)



Figure 9. Number of FGMRES-iterations (colour code) to solve the $\mathscr{H}$-BE equation (5) for interface displacements in the iterative Dirichlet-Neumann algorithm as a function of the frequency and the iteration step $k$, for a spherical cavity embedded in a layered space with (a) $\alpha=1 / 2$, (b) $\alpha=1$ and (c) $\alpha=2$.

Figure 12 shows the real and imaginary part of the vertical displacement $\hat{u}_{z}(\mathbf{x}, \omega)$ at the center of the foundation, calculated with both coupling procedures; a perfect agreement between the results is observed. The peak at $20 \mathrm{~Hz}$ is caused by resonance of the foundation on the layered halfspace. The iterative Neumann-Dirichlet is unable, however, to determine the static solution, and the frequency sweep is therefore performed from high to low frequencies. The vertical displacement of the foundation and the surrounding soil is shown in figures $13 \mathrm{a}$ and $13 \mathrm{~b}$ at $25 \mathrm{~Hz}$ and $100 \mathrm{~Hz}$, respectively. While the wave fronts at the surface of the soil remain almost perfectly cylindrical at $25 \mathrm{~Hz}$, this is no longer the case at $100 \mathrm{~Hz}$ due to the dynamic interaction between the foundation and the soil.

Figure 14a shows the number of FGMRES-iterations as a function of the frequency and the iteration step $k$ for the sequential Neumann-Dirichlet algorithm, in case no initial guess is provided to the FGMRES solver. The number of FGMRES-iterations remains almost independent of the 


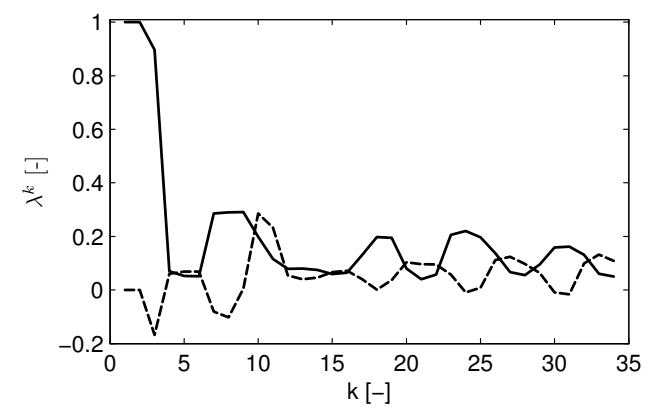

Figure 10. Real (solid line) and imaginary (dashed line) part of the Aitken relaxation parameter $\lambda^{k}$ at $100 \mathrm{~Hz}$ in the sequential Neumann-Dirichlet iterative algorithm, for a spherical cavity embedded in a layered space with $\alpha=1$.

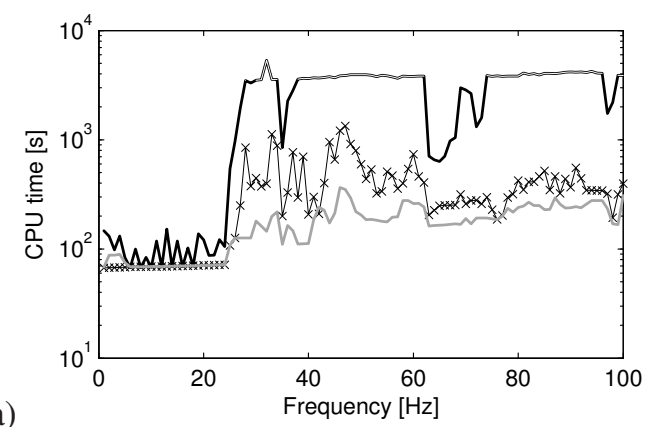

(a)

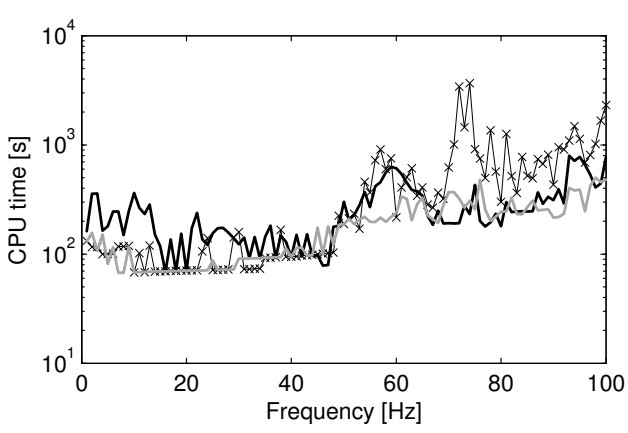

(b)

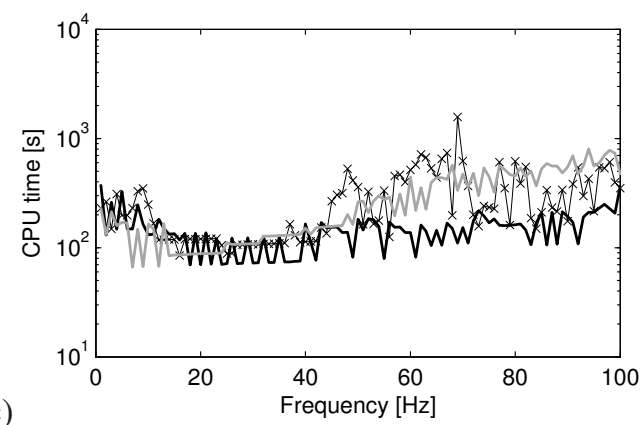

Figure 11. CPU time required in the parallel Neumann-Neumann algorithm in case the displacement discrepancy is imposed on the BE subdomain (solid black line), on the FE subdomain (solid grey line), and in case the relaxation procedure corresponding to equation (17) is employed (crosses), for a spherical cavity embedded in a layered space with (a) $\alpha=1 / 2$, (b) $\alpha=1$ and (c) $\alpha=2$. The lines are only drawn if convergence is achieved. 

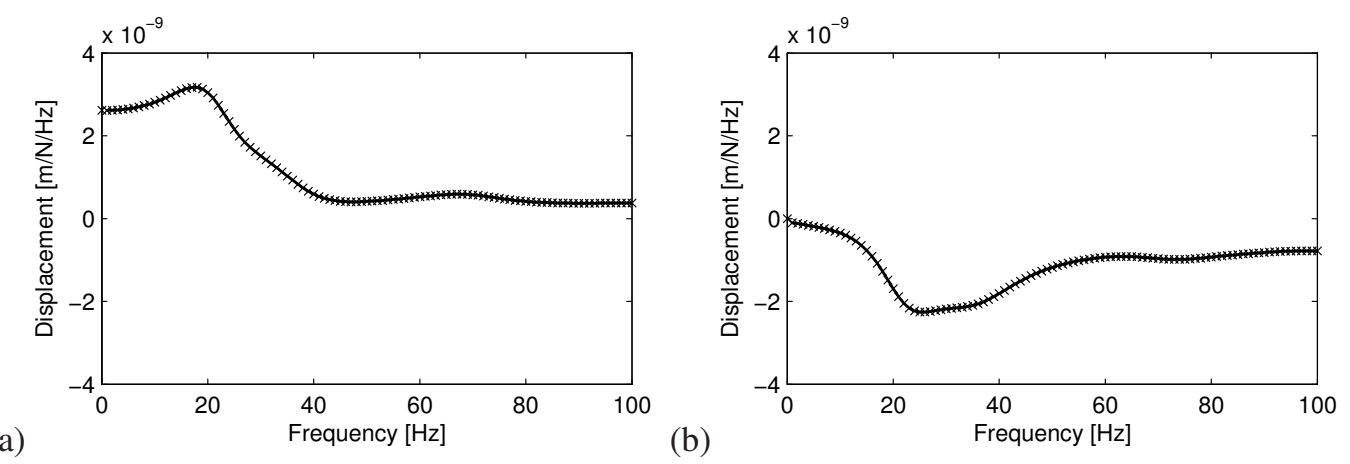

Figure 12. (a) Real and (b) imaginary part of the vertical displacement $\hat{u}_{z}(\mathbf{x}, \omega)$ at the center of a flexible surface foundation on a layered halfspace excited by a unit harmonic vertical point load at its center. The solution of the iterative Neumann-Dirichlet algorithm (solid line) is compared to the solution of the monolithic coupling procedure (crosses).

(a)

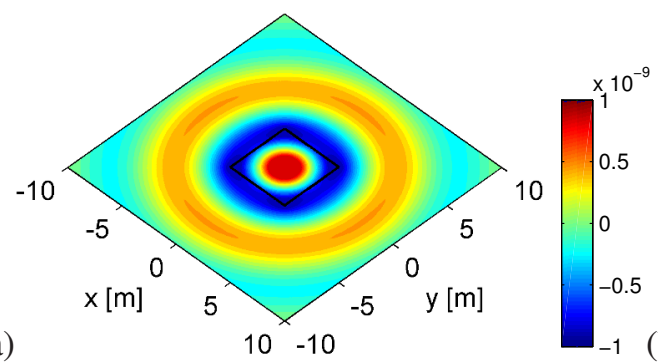

(b)

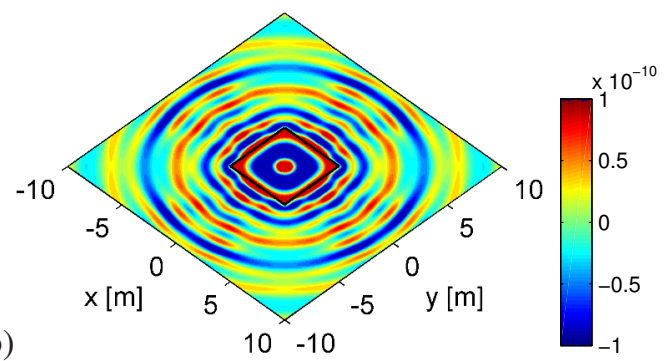

Figure 13. Real part of the vertical displacement $\hat{u}_{z}(\mathbf{x}, \omega)$ of the foundation and the soil for a flexible surface foundation on a layered halfspace excited by a unit harmonic vertical point load at its center at (a) $25 \mathrm{~Hz}$ and

(b) $100 \mathrm{~Hz}$.

iteration step $k$ if this strategy is applied. It is illustrated in figure $14 \mathrm{~b}$ that imposing the relaxed interaction forces obtained in step $k-1$ as initial guess in the FGMRES solver of step $k$ is clearly beneficial, as the number of FGMRES-iterations in subsequent steps is strongly reduced. Using a start vector in the first Neumann-Dirichlet iteration corresponding to the converged solution of the previous frequency step is also advantageous; this results in a lower numbers of iteration steps per frequency. The peaks at $40 \mathrm{~Hz}$ and $62 \mathrm{~Hz}$ in figure 14 correspond to natural frequencies of the free foundation. Applying Neumann boundary conditions to the FE subdomain near a natural frequency results in large displacements, and additional iterations are hence required to balance the resulting mismatch of displacements and tractions on the interface. It should be emphasized that these natural frequencies differ from the resonance of the foundation on the soil at $20 \mathrm{~Hz}$ (apparent in figure 12), which is due to the dynamic SSI.

\section{APPLICATION: SUBGRADE STIFFENING AS A MITIGATION MEASURE FOR RAILWAY INDUCED VIBRATIONS}

The numerical examples considered in section 4 demonstrate the validity and efficiency of the presented coupling algorithms. These methodologies enable the fast evaluation of large coupled FE- $\mathscr{H}$-BE models, providing the possibility to analyze complex problems of dynamic SSI. The verification examples discussed in section 4 involve only a limited amount of degrees of freedom. An engineering application is therefore considered in this section to illustrate the applicability of coupled FE- $\mathscr{H}$-BE models to large scale problems. 
(a)
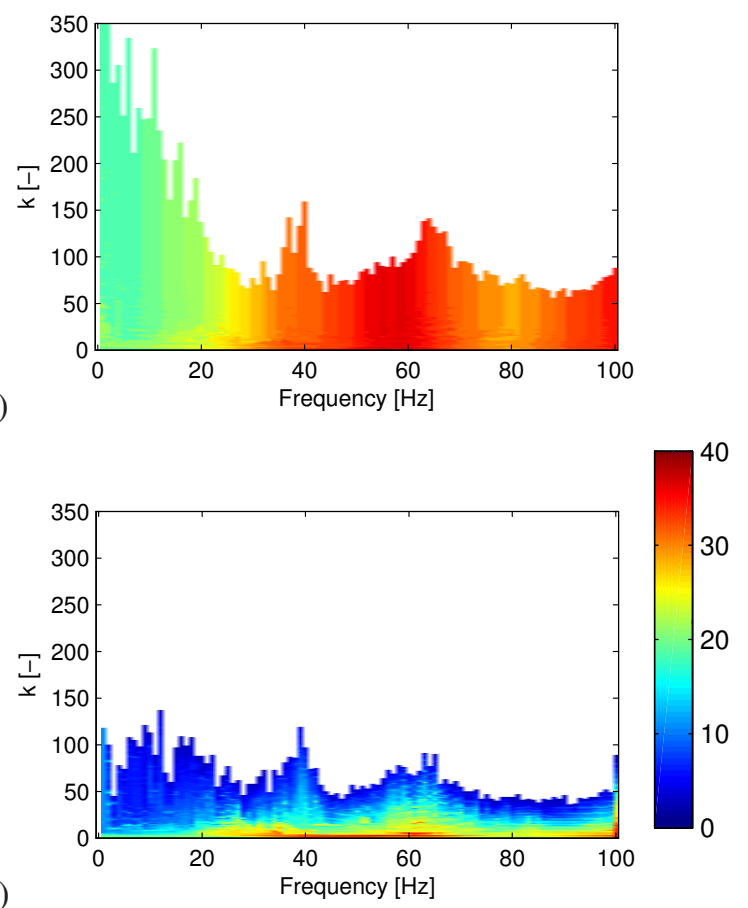

Figure 14. Number of FGMRES-iterations (colour code) to solve the $\mathscr{H}$-BE equation (5) for tractions in the iterative Neumann-Dirichlet algorithm as a function of the frequency and the iteration step $k$, for a flexible surface foundation on a layered halfspace. The numbers are shown (a) in case no initial guess is provided to the FGMRES solver, or (b) in case the relaxed tractions in iteration $k-1$ are used as an initial guess in iteration $k$, while the converged solution at a particular frequency is used as an initial guess for the first iteration at the subsequent frequency.

The application is related to the mitigation of railway induced vibrations, which can lead to annoyance in the built environment. An important category of vibration reduction measures are interventions in the propagation path between source (railway track) and receiver (building), such as vibration isolation screens [47], buried wall barriers [48], and wave impeding blocks [49]. Recently, the vibration reduction efficiency of subgrade stiffening has been investigated [50], where a block of stiffened soil is included in the transmission path, e.g. by means of jet grouting (figure 15a). In order reduce the computational effort, the geometry of these mitigation measures is often assumed to be invariant in the longitudinal direction, allowing for the application of an efficient two-and-ahalf-dimensional (2.5D) approach, where a Fourier transform of the longitudinal coordinate allows representing the $3 \mathrm{D}$ response on a $2 \mathrm{D}$ mesh [51]. In practice, the construction of a jet grouting wall is limited to finite dimensions, however, and the assumption of longitudinal invariance of the geometry is therefore not fulfilled (figure 15b). Accounting for the finite length requires the solution of a full 3D dynamic SSI problem, which is computationally very demanding if a classical BE formulation is used; the $\mathrm{FE}-\mathscr{H}-\mathrm{BE}$ methods proposed in this paper are much more appropriate to solve this problem.

The vibration reduction efficiency of a block of stiffened soil of finite length embedded in a halfspace is investigated in this section. A case study involving a block with a width $b=2 \mathrm{~m}$, a depth $h=2 \mathrm{~m}$, a length $L_{y}$, and situated at a distance $d=4 \mathrm{~m}$ from the $y$-axis is considered (figure 15). In order to facilitate physical interpretation, the soil is assumed to be homogeneous, and an incident wavefield is generated by the application of a unit vertical harmonic point load at the origin of the coordinate system, rather than considering the passage of a train. The halfspace is characterized by a shear wave velocity $C_{\mathrm{s}}=200 \mathrm{~m} / \mathrm{s}$, a dilatational wave velocity $C_{\mathrm{p}}=400 \mathrm{~m} / \mathrm{s}$, a density 
(a)

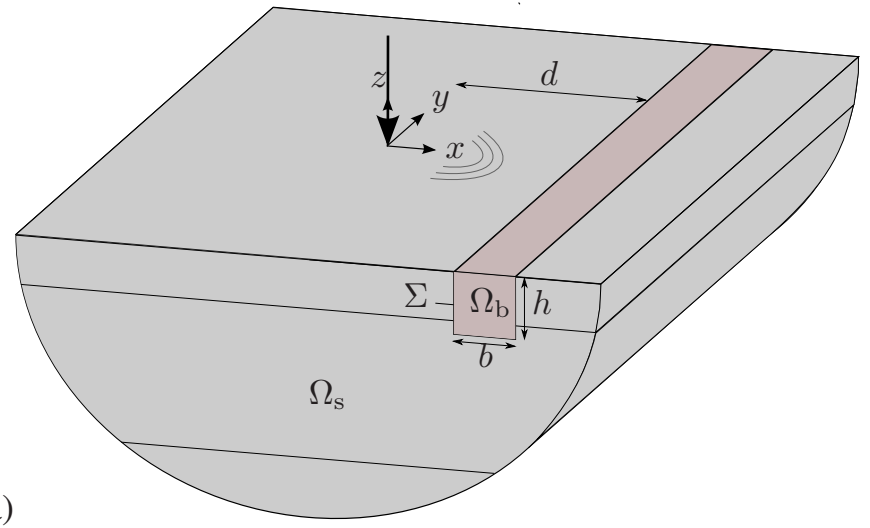

(b)

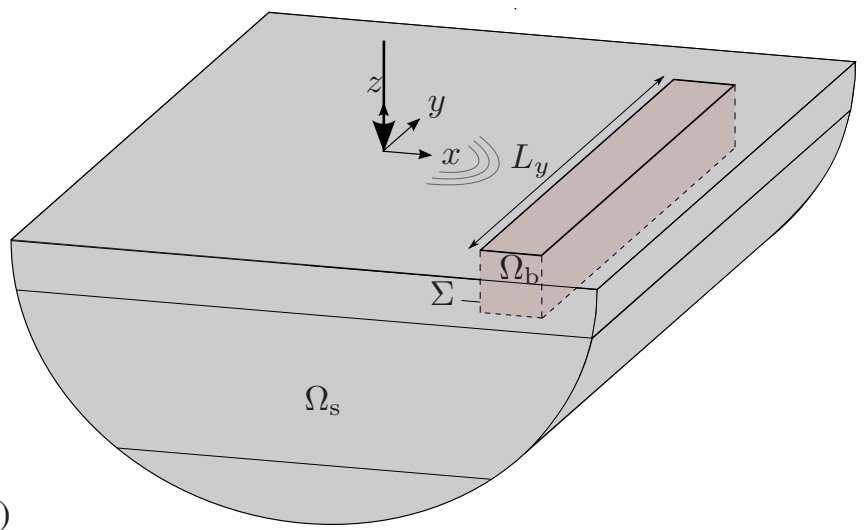

Figure 15. A block of stiffened soil $\Omega_{\mathrm{b}}$ incorporated in the soil domain $\Omega_{\mathrm{s}}$, (a) assuming longitudinal invariance of the geometry or (b) accounting for the finite length $L_{y}$.

$\rho=2000 \mathrm{~kg} / \mathrm{m}^{3}$, and material damping ratios $\beta_{\mathrm{s}}=\beta_{\mathrm{p}}=0.025$ in both deviatoric and volumetric deformation. The Rayleigh wave velocity $C_{\mathrm{R}}=186.7 \mathrm{~m} / \mathrm{s}$ is frequency independent due to its nondispersive behaviour in a homogeneous halfspace [52]. The block of stiffened soil has a shear wave velocity $C_{\mathrm{s}}=550 \mathrm{~m} / \mathrm{s}$, a dilatational wave velocity $C_{\mathrm{p}}=950 \mathrm{~m} / \mathrm{s}$, a density $\rho=2000 \mathrm{~kg} / \mathrm{m}^{3}$, and material damping ratios $\beta_{\mathrm{s}}=\beta_{\mathrm{p}}=0.050$.

The block of stiffened soil is discretized by means of eight-node solid elements, which are coupled to a conforming mesh of four-node quadrilateral boundary elements for the surrounding soil domain. Halfspace Green's functions are employed in the $\mathscr{H}$-BE formulation. The numerical analysis is performed for a block of stiffened soil with a length of $15 \mathrm{~m}, 30 \mathrm{~m}$, and $60 \mathrm{~m}$; the properties of the resulting FE and BE discretizations are listed in table I. As there exists a strong stiffness contrast between the FE and $\mathscr{H}$-BE subdomain, an iterative Neumann-Dirichlet algorithm is employed for the solution of the $3 \mathrm{D}$ coupled $\mathrm{FE}-\mathscr{H}$-BE problem.

\begin{tabular}{ccccccc}
\hline $\begin{array}{c}L_{y} \\
{[\mathrm{~m}]}\end{array}$ & $\begin{array}{c}\text { \# FE elements } \\
{[-]}\end{array}$ & $\begin{array}{c}\text { \# FE nodes } \\
{[-]}\end{array}$ & $\begin{array}{c}\text { \# FE DOFs } \\
{[-]}\end{array}$ & $\begin{array}{c}\text { \# BE elements } \\
{[-]}\end{array}$ & $\begin{array}{c}\text { \# BE nodes } \\
{[-]}\end{array}$ & $\begin{array}{c}\text { \# BE DOFs } \\
{[-]}\end{array}$ \\
\hline 15 & 3840 & 4941 & 14823 & 1568 & 1637 & 4911 \\
30 & 7680 & 9801 & 29403 & 3008 & 3137 & 9411 \\
60 & 15360 & 19521 & 58563 & 5888 & 6137 & 18411 \\
\hline
\end{tabular}

Table I. Properties of the FE and BE discretizations of a block of stiffened soil with a length of $15 \mathrm{~m}, 30 \mathrm{~m}$, and $60 \mathrm{~m}$. 
Figures 16-18a show the real part of the vertical displacement $\hat{u}_{z}(\mathbf{x}, \omega)$ in the reference case (i.e. without subgrade stiffening) at a frequency of $15 \mathrm{~Hz}, 30 \mathrm{~Hz}$, and $60 \mathrm{~Hz}$, respectively. The wavefield in the soil is characterized by cylindrical wave fronts with a Rayleigh wavelength $\lambda_{\mathrm{R}}(\omega)=2 \pi C_{\mathrm{R}} / \omega$ equal to $12.45 \mathrm{~m}, 6.22 \mathrm{~m}$, and $3.11 \mathrm{~m}$, respectively. The real part of the vertical displacement $\hat{u}_{z}(\mathbf{x}, \omega)$ in case an infinitely long block of stiffened soil is embedded in the halfspace is shown in figures $16-18 \mathrm{e}$. These results are obtained by means of a $2.5 \mathrm{D}$ coupled FE-BE calculation based on the assumption of longitudinal invariance of the geometry. At $15 \mathrm{~Hz}$, the wavefield in the soil is only slightly affected by the presence of the block of stiffened soil (figure 16e), while a larger influence is observed at higher frequencies (figures 17-18e). The vibration reduction efficiency in each of these cases is characterized through the corresponding vertical insertion loss $\widehat{\mathrm{IL}}_{z}(\mathbf{x}, \omega)$ :

$$
\widehat{\mathrm{IL}}_{z}(\mathbf{x}, \omega)=20 \log _{10} \frac{\left|\hat{u}_{z}^{\mathrm{ref}}(\mathbf{x}, \omega)\right|}{\left|\hat{u}_{z}(\mathbf{x}, \omega)\right|}
$$

Positive values of the insertion loss indicate a reduction of the vertical free field vibrations. It has been demonstrated in [50] that the wave impeding effect depends on the relation between the Rayleigh wavelength in the soil and the free bending wavelength in the block of stiffened soil, as the transmission of plane waves in the soil with a longitudinal wavelength smaller than the bending wavelength is hindered. The mitigation measure is only effective above a critical frequency depending on the stiffness contrast between the soil and the block of stiffened soil. The guideline formulated in [50] states that the critical frequency can be estimated as $\frac{C_{\mathrm{R}}^{2}}{2 \pi h} \sqrt{\frac{12 \rho}{E}}=12 \mathrm{~Hz}$, where $C_{\mathrm{R}}$ is the Rayleigh wave velocity of the halfspace and $h, \rho$, and $E$ are characteristics of the block of stiffened soil. The frequencies under concern are all above this critical frequency, and a wave impeding effect is hence observed in figures 16-18e. The area where vibration levels are significantly reduced clearly depends on the frequency, however, and a critical angle delimiting this area can be distinguished. An analytical expression for the latter is also given in [50].

Figures 16-18b-d show the real part of the vertical displacement $\hat{u}_{z}(\mathbf{x}, \omega)$ and the insertion loss $\widehat{\mathrm{IL}}_{z}(\mathbf{x}, \omega)$ in case a block of stiffened soil is included over a finite length of $15 \mathrm{~m}, 30 \mathrm{~m}$, and $60 \mathrm{~m}$ at a frequency of $15 \mathrm{~Hz}, 30 \mathrm{~Hz}$, and $60 \mathrm{~Hz}$, respectively, computed by means of the 3D coupled FE$\mathscr{H}$-BE iterative Neumann-Dirichlet method. At $15 \mathrm{~Hz}$, neither a length of $15 \mathrm{~m}$ or $30 \mathrm{~m}$ is sufficient to create a wave impeding effect similar to the case where the block is assumed to be of infinite length; a block length of $60 \mathrm{~m}$, however, does result in a comparable insertion loss. The wavefield in the soil and the insertion loss at $30 \mathrm{~Hz}$ resembles the result of the $2.5 \mathrm{D}$ calculation for a block with a length of $30 \mathrm{~m}$ or larger, while a good correspondence with the latter is achieved for all lengths at a frequency of $60 \mathrm{~Hz}$.

The observations in figures 16-18 can be interpreted by comparing the length $L_{y}$ of the block to the free bending wavelength $\lambda_{\mathrm{b}}(\omega)$ of an infinitely long beam. The latter yields $\lambda_{\mathrm{b}}(\omega)=$ $2 \pi\left(\frac{E h^{2}}{12 \rho \omega^{2}}\right)^{1 / 4}$ for an Euler-Bernoulli beam and is equal to $14.50 \mathrm{~m}, 10.25 \mathrm{~m}$, and $7.25 \mathrm{~m}$ at $15 \mathrm{~Hz}$, $30 \mathrm{~Hz}$, and $60 \mathrm{~Hz}$, respectively. This indicates that the block should be approximately twice as long as the free bending wavelength $\lambda_{\mathrm{b}}(\omega)$ in order to create an efficient wave impeding barrier; a vibration reduction efficiency comparable to that of a block of infinite length is then achieved. The finite block is only able, however, to impede the transmission of that part of the wavefield that actually impinges on the block, which is clearly visible for a block of $15 \mathrm{~m}$ (figures 16-18b). Furthermore, diffraction occurs at the extremities of the finite block.

\section{CONCLUSIONS}

In this paper, the coupling of FE and $\mathscr{H}$-BE methods has been discussed, illustrating that a subdomain approach is well suited to efficiently solve dynamic SSI problems in the frequency domain. The application of $\mathscr{H}$-matrices enables the fast evaluation of large BE models, and the incorporation of Green's functions for a layered halfspace avoids the need to discretize the free 
(a)

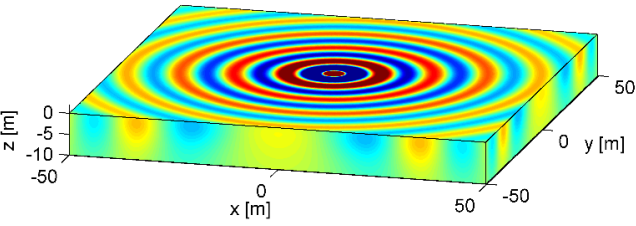

b)
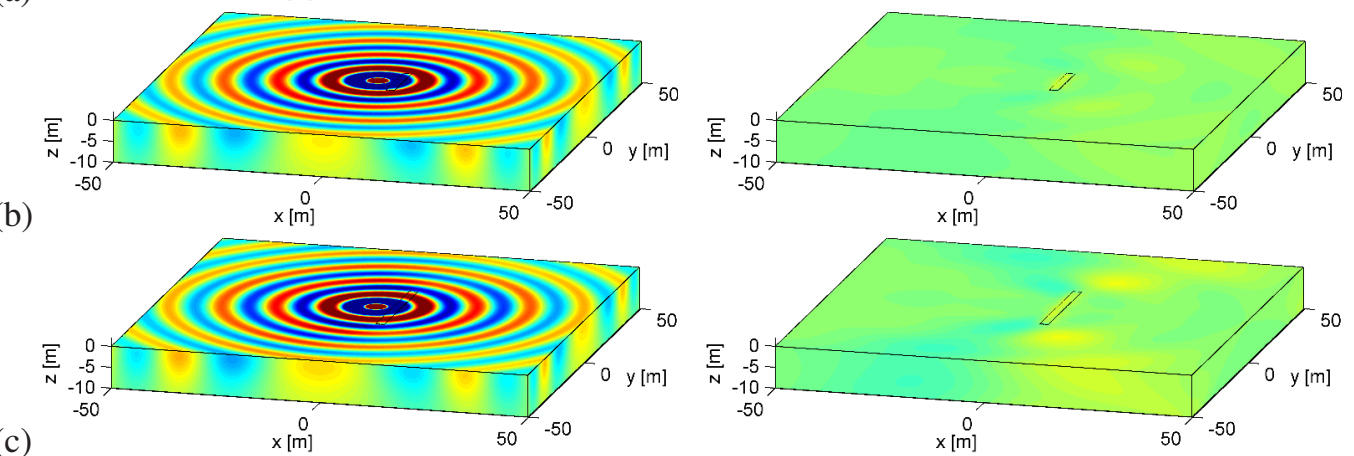

(c)

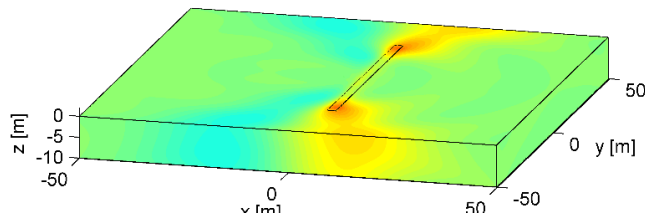

(d)
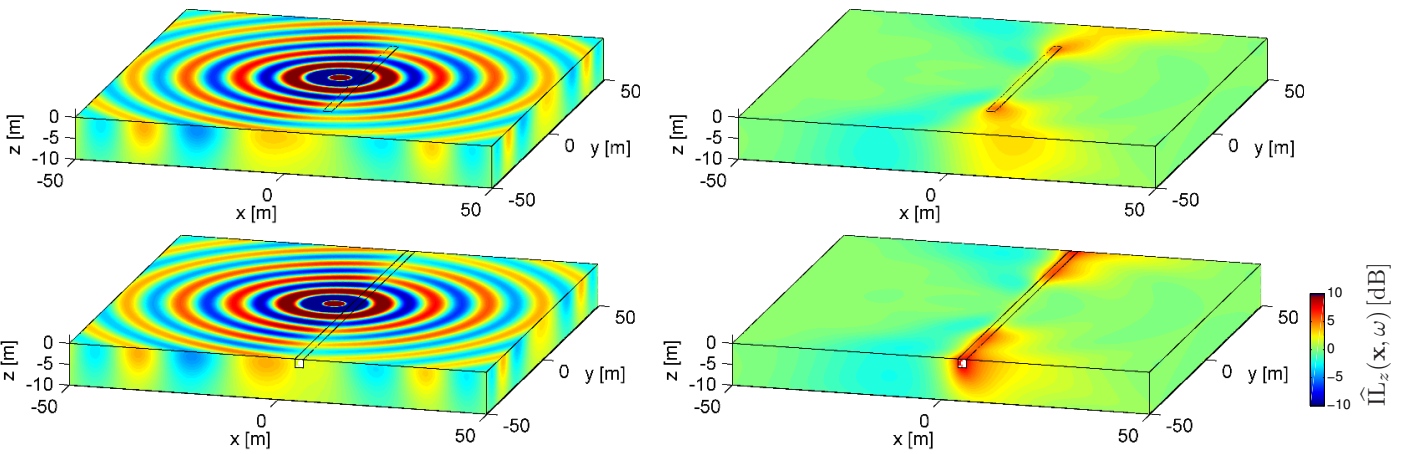

(e)

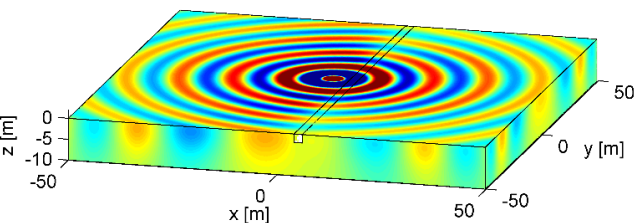

Figure 16. Real part of the vertical displacement $\hat{u}_{z}(\mathbf{x}, \omega)$ due to harmonic excitation at $15 \mathrm{~Hz}$ (a) in the reference case and in case a block of stiffened soil is included with a length (b) $L_{y}=15 \mathrm{~m}$, (c) $L_{y}=30 \mathrm{~m}$, (d) $L_{y}=60 \mathrm{~m}$, and (e) $L_{y}=\infty$ (left hand side). The corresponding insertion loss $\widehat{\mathrm{IL}}_{z}(\mathbf{x}, \omega)$ is shown at the right hand side.

surface and the layer interfaces in the modelling of visco-elastodynamic wave propagation in a stratified medium, as illustrated in subsection 4.2.

Direct, iterative and monolithic coupling strategies have been considered in this paper and the numerical verification of each algorithm is presented. An assessment of the computational performance reveals that the direct coupling approach is the least efficient, as the assembly of a dynamic soil stiffness matrix requires the solution of a large amount of $\mathscr{H}-\mathrm{BE}$ equations. Iterative coupling procedures are more efficient, provided that suitable boundary conditions are applied to each subdomain. It is demonstrated that sequential iterative algorithms should be preferred if there exists a strong stiffness contrast between the FE and $\mathscr{H}$-BE subdomain, with Neumann boundary conditions to be imposed on the most stiff subdomain. The application of Aitken's $\Delta^{2}$-method for the determination of a proper interface relaxation parameter ensures and speeds up the convergence of these sequential algorithms. Parallel iterative algorithms provide a valuable alternative for cases where an a priori estimation of the flexibility of each subdomain is not evident and an appropriate novel relaxation procedure has been proposed for these algorithms. An efficient combination of FE and $\mathscr{H}-\mathrm{BE}$ models can also be achieved by means of a monolithic coupling scheme, although the convergence in the examples considered turns out to be relatively slow. This might be improved by the incorporation of an enhanced preconditioner, which is a subject for further research.

Finally, an engineering application related to the mitigation of railway induced vibrations has been presented to illustrate the applicability of coupled FE- $\mathscr{H}$-BE methods to large scale problems. 
(a)



(b)
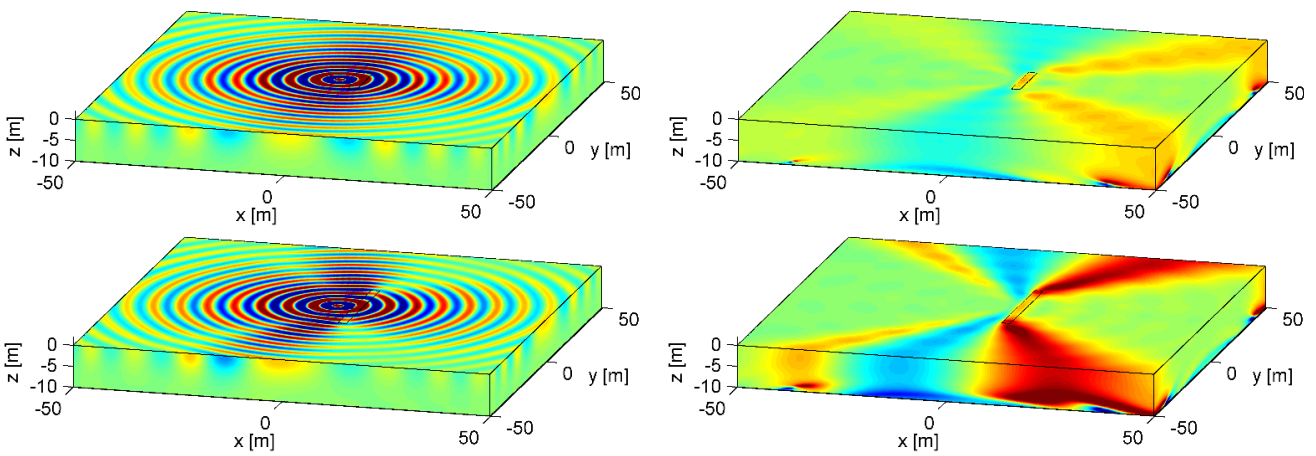

(c)
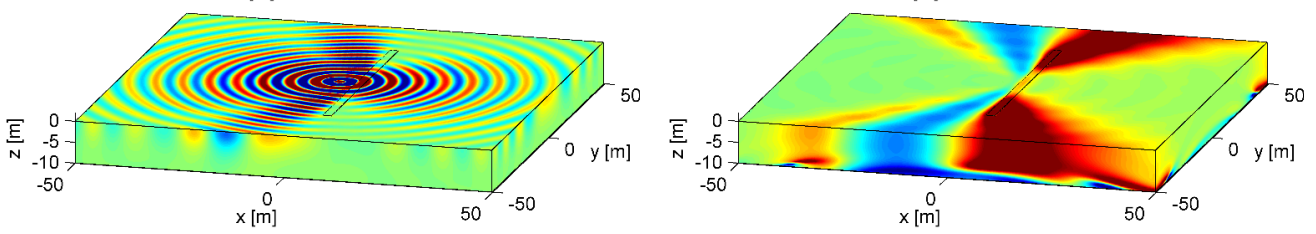

(d)
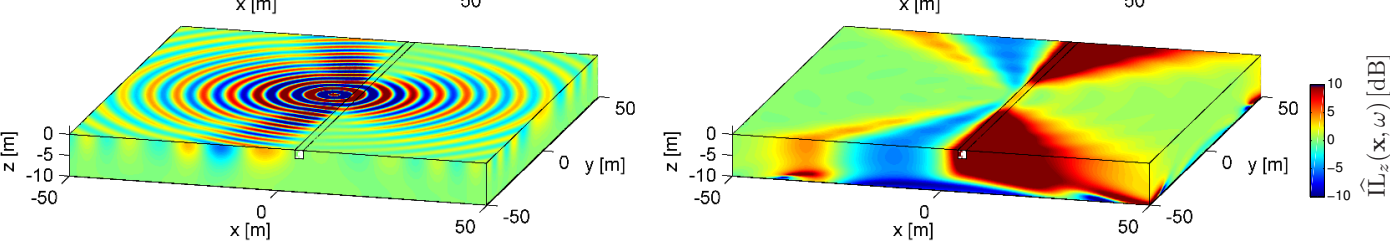

Figure 17. Real part of the vertical displacement $\hat{u}_{z}(\mathbf{x}, \omega)$ due to harmonic excitation at $30 \mathrm{~Hz}$ (a) in the reference case and in case a block of stiffened soil is included with a length (b) $L_{y}=15 \mathrm{~m}$, (c) $L_{y}=30 \mathrm{~m}$, (d) $L_{y}=60 \mathrm{~m}$, and (e) $L_{y}=\infty$ (left hand side). The corresponding insertion loss $\widehat{\mathrm{IL}}_{z}(\mathbf{x}, \omega)$ is shown at the right hand side.

The vibration reduction efficiency of a block of stiffened soil in a homogeneous halfspace has been assessed through 3D calculations, indicating that the length of the block should be approximately twice the free bending wavelength of an infinitely long beam in order to create an efficient wave impeding barrier.

\section{ACKNOWLEDGEMENTS}

The first author is a doctoral fellow and the second author is a postdoctoral fellow of the Research Foundation Flanders (FWO). The results presented in section 5 have been obtained within the frame of the EU FP7 project RIVAS (Railway Induced Vibration Abatement Solutions) [53] under grant agreement No. 265754. The financial support is gratefully acknowledged. 
(a)
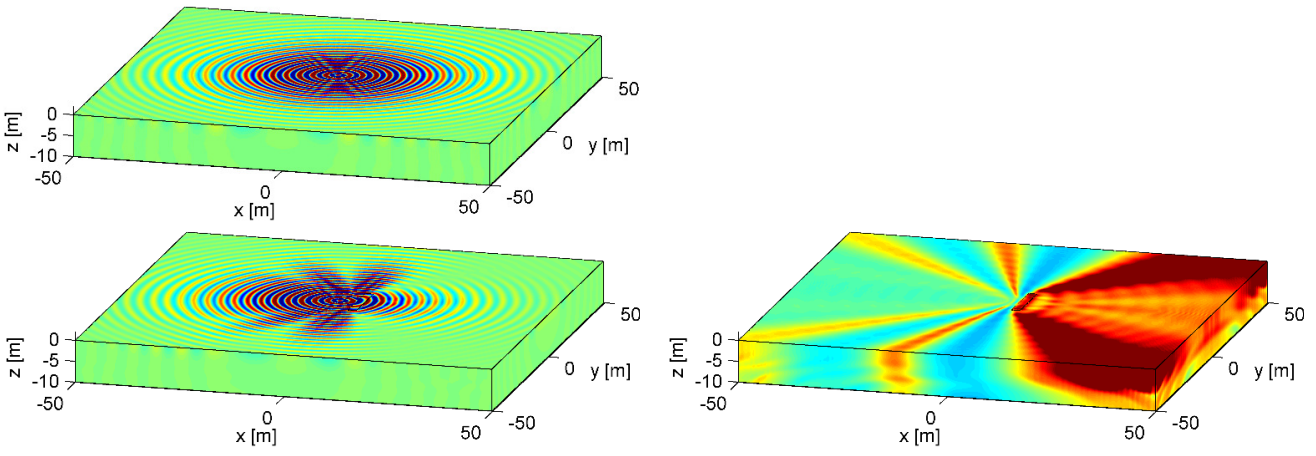

(b)
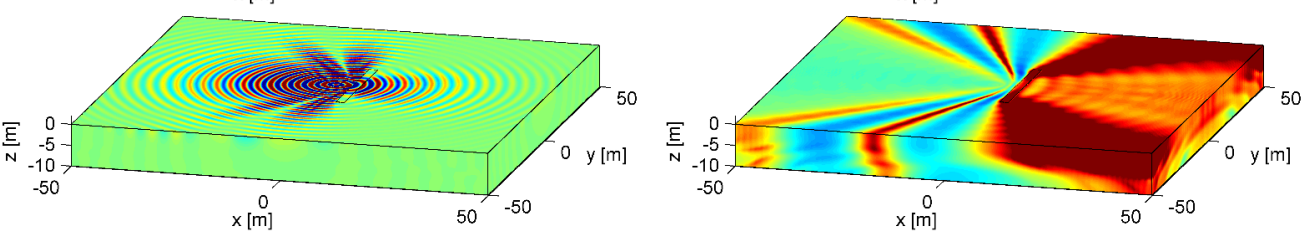

(c)

$$
x[\stackrel{0}{m}]
$$
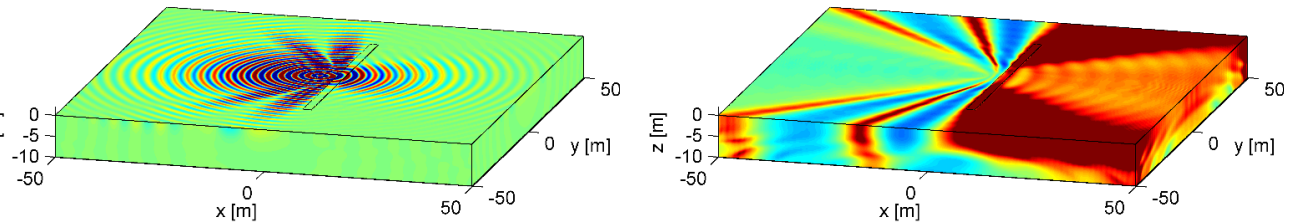

(d)
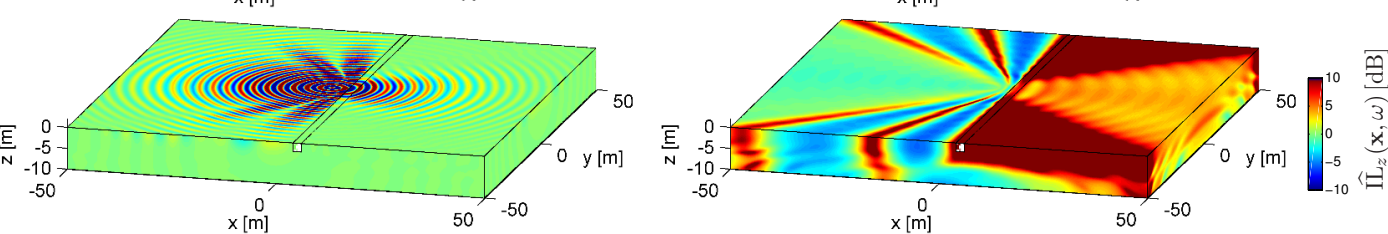

Figure 18. Real part of the vertical displacement $\hat{u}_{z}(\mathbf{x}, \omega)$ due to harmonic excitation at $60 \mathrm{~Hz}$ (a) in the reference case and in case a block of stiffened soil is included with a length (b) $L_{y}=15 \mathrm{~m}$, (c) $L_{y}=30 \mathrm{~m}$, (d) $L_{y}=60 \mathrm{~m}$, and (e) $L_{y}=\infty$ (left hand side). The corresponding insertion loss $\widehat{\mathrm{IL}}_{z}(\mathbf{x}, \omega)$ is shown at the right hand side. 


\section{REFERENCES}

1. Beskos D, Krauthammer T, Vardoulakis I ( (eds.)). Dynamic soil-structure interaction, A.A. Balkema, 1984.

2. Von Estorff O, Hagen C. Iterative coupling of FEM and BEM in 3D transient elastodynamics. Engineering Analysis with Boundary Elements 2005; 29:775-787.

3. Clouteau D, Elhabre M, Aubry D. Periodic BEM and FEM-BEM coupling: application to seismic behaviour of very long structures. Computational Mechanics 2000; 25:567-577.

4. Zienkiewicz O, Kelly D, Bettes P. The coupling of the finite element method and boundary solution procedures. International Journal for Numerical Methods in Engineering 1977; 11:355-375.

5. Hsiao G. The coupling of boundary element and finite element methods. Zeitschrift für Angewandte Mathematik und Mechanik 1990; 70:493-503.

6. Costabel M, Stephan E. Coupling of finite and boundary element methods for an elastoplastic interface problem. SIAM Journal on Numerical Analysis 1990; 27(5):1212-1226.

7. Elleithy W, Al-Gahtani HJ, El-Gebeily M. Iterative coupling of BE and FE methods in elastostatics. Engineering Analysis with Boundary Elements 2001; 25:685-695.

8. Margonari M, Bonnet M. Fast multipole method applied to elastostatic BEM-FEM coupling. Computers and Structures 2004; 83(10-11):700-717.

9. Rüberg T, Schanz M. Coupling finite and boundary element methods for static and dynamic elastic problems with non-conforming interfaces. Computer Methods in Applied Mechanics and Engineering 2008; 198(3-4):449-458.

10. Chaillat S, Bonnet M, Semblat JF. A multi-level fast multipole BEM for 3-D elastodynamics in the frequency domain. Computer Methods in Applied Mechanics and Engineering 2008; 197(49-50):4233-4249.

11. Hackbusch W. A sparse matrix arithmetic based on $\mathscr{H}$-matrices. Part I: Introduction to $\mathscr{H}$-matrices. Computing 1999; 62(2):89-108.

12. Coulier P, François S, Lombaert G, Degrande G. The numerical prediction of railway induced vibrations in an urban environment. Proceedings of the 10th World Congress on Computational Mechanics WCCM 2012, Pimenta P, Campello E (eds.), Sao Paulo, Brazil, 2012. CD-ROM.

13. Kausel E, Roësset J. Stiffness matrices for layered soils. Bulletin of the Seismological Society of America 1981; 71(6):1743-1761.

14. Kausel E. Fundamental solutions in elastodynamics: a compendium. Cambridge University Press: New York, 2006.

15. Grasso E. Modelling visco-elastic seismic wave propagation: a fast-multipole boundary element method and its coupling with finite elements. PhD Thesis, Université Paris-Est 2012.

16. Soares Jr D, Godinho L. An optimized BEM-FEM iterative coupling algorithm for acoustic-elastodynamic interaction analyses in the frequency domain. Computers and Structures 2012; 106-107:68-80.

17. Aitken A. Studies in practical mathematics II: The evaluation of the latent roots and latent vectors of a matrix. Proceedings of the Royal Society of Edinburgh 1937; 57:269-304.

18. Hübner B, Walhorn E, Dinkler D. A monolithic approach to fluid-structure interaction using space-time finite elements. Computer Methods in Applied Mechanics and Engineering 2004; 193(23-26):2087-2104.

19. Michler C, Hulshoff S, van Brummelen E, de Borst R. A monolithic approach to fluid-structure interaction. Computers and Fluids 2004; 33:839-848.

20. Zienkiewicz O, Taylor R. The finite element method, Volume 1: the basis. Fifth edn., Butterworth-Heinemann: Oxford, United Kingdom, 2000.

21. Bonnet M. Boundary integral equation methods for solids and fluids. John Wiley and Sons: Chichester, United Kingdom, 1995

22. Rizzo F, Shippy D, Rezayat M. A boundary integral equation method for radiation and scattering. International Journal for Numerical Methods in Engineering 1985; 21:115-129.

23. Aubry D, Clouteau D. A regularized boundary element method for stratified media. Proceedings of the First International Conference on Mathematical and Numerical Aspects of Wave Propagation Phenomena, Cohen G, Halpern L, Joly P (eds.), SIAM, Philadephia: Strasbourg, France, 1991; 660-668.

24. Schenck H. Improved integral formulation for acoustic radiation problems. Journal of the Acoustical Society of America $1968 ; 44: 41-58$

25. Grasedyck L, Hackbusch W. Construction and arithmetics of $\mathscr{H}$-matrices. Computing 2003; 70:295-334.

26. Bebendorf M. Hierarchical Matrices: A Means to Efficiently Solve Elliptic Boundary Value Problems. 1st edn., Springer Publishing Company, 2008.

27. Rjasanow S, Steinbach O. The Fast Solution of Boundary Integral Equations (Mathematical and Analytical Techniques with Applications to Engineering). Springer-Verlag New York, 2007.

28. Bebendorf M, Rjasanow S. Adaptive low-rank approximation of collocation matrices. Computing 2003; 70:1-24.

29. Milazzo A, Benedetti I, Aliabadi M. Hierarchical fast BEM for anisotropic time-harmonic 3-D elastodynamics. Computers and Structures 2012; 96-97:9-24.

30. Saad Y, Schultz M. GMRES: a generalized minimal residual algorithm for solving nonsymmetric linear systems. SIAM Journal on Scientific and Statistical Computing 1986; 7(3):856-869.

31. Bebendorf M. Hierarchical $L U$ decomposition based preconditioners for BEM. Computing 2005; 74:225-247.

32. Chaillat S, Semblat JF, Bonnet M. A preconditioned 3-D multi-region fast multipole solver for seismic wave propagation in complex geometries. Communications in Computational Physics 2012; 11(2):594-609.

33. Saad Y. A flexible inner-outer preconditioned GMRES algorithm. SIAM Journal on Scientific and Statistical Computing 1993; 14(2):461-469.

34. Von Estorff O, Kausel E. Coupling of boundary and finite elements for soil-structure interaction. Earthquake Engineering and Structural Dynamics 1989; 18:1065-1075.

35. Clouteau D, Arnst M, Al-Hussaini T, Degrande G. Freefield vibrations due to dynamic loading on a tunnel embedded in a stratified medium. Journal of Sound and Vibration 2005; 283(1-2):173-199. URL http://dx.doi.org/10.1016/j.jsv.2004.04.010. 
36. Von Estorff O, Firuziaan M. Coupled BEM/FEM approach for nonlinear soil/structure interaction. Engineering Analysis with Boundary Elements 2000; 24:605-621.

37. François S, Masoumi H, Degrande G. An iterative coupled boundary-finite element method for the dynamic response of structures. Proceedings of ISMA2006 International Conference on Noise and Vibration Engineering, Sas P, De Munck M (eds.), Leuven, Belgium, 2006; 1701-1716.

38. Soares Jr D, Godinho L, Pereira A, Dors C. Frequency domain analysis of acoustic wave propagation in heterogeneous media considering iterative coupling procedures between the method of fundamental solutions and Kansa's method. International Journal for Numerical Methods in Engineering 2012; 89(7):914-938. URL http://dx.doi.org/10.1002/nme.3274.

39. Elleithy W, Tanaka M. Interface relaxation algorithms for BEM-BEM coupling and FEM-BEM coupling. Computer Methods in Applied Mechanics and Engineering 2003; 192:2977-2992.

40. Hagen C. Wechselwirkungen zwischen Bauwerk, Boden und Fluid unter transienter Belastung. PhD Thesis, Technischen Universität Hamburg-Harburg 2005.

41. Mok D, Wall W, Ramm E. Accelerated iterative substructuring schemes for instationary fluid-structure interaction. Computational Fluid and Solid Mechanics, Bathe K (ed.). Elsevier, 2001; 1325-1328.

42. Küttler U, Wall W. Fixed-point fluid-structure interaction solvers with dynamic relaxation. Computational Mechanics 2008; 43(1):61-72.

43. Irons B, Tuck R. A version of the Aitken accelerator for computer iteration. International Journal for Numerical Methods in Engineering 1969; 1(3):275-277.

44. Chaillat S, Bonnet M, Semblat JF. A new fast multi-domain BEM to model seismic wave propagation and amplification in 3D geological structures. Geophysical Journal International 2009; 177:509-531

45. Langer U, Pusch D. Data-sparse algebraic multigrid methods for large scale boundary element equations. Applied Numerical Mathematics 2005; 54(3-4):406-424.

46. Schevenels M, François S, Degrande G. EDT: An ElastoDynamics Toolbox for MATLAB. Computers \& Geosciences 2009; 35(8):1752-1754. URL http: / / dx. doi.org/10.1016/j . cageo.2008.10.012.

47. Woods R. Screening of surface waves in soils. Journal of the Soil Mechanics and Foundation Division, Proceedings of the ASCE 1968; 94(SM4):951-979.

48. Andersen L, Nielsen S. Reduction of ground vibration by means of barriers or soil improvement along a railway track. Soil Dynamics and Earthquake Engineering 2005; 25:701-716.

49. Sheng X, Jones C, Thompson D. Modelling ground vibrations from railways using wavenumber finite- and boundary-element methods. Proceedings of the Royal Society A - Mathematical, Physical and Engineering Sciences 2005; 461:2043-2070.

50. Coulier P, François S, Degrande G, Lombaert G. Subgrade stiffening next to the track as a wave impeding barrier for railway induced vibrations. Soil Dynamics and Earthquake Engineering 2013; 48:119-131. URL http://dx.doi.org/10.1016/j.soildyn.2012.12.009.

51. François S, Schevenels M, Lombaert G, Galvín P, Degrande G. A 2.5D coupled FE-BE methodology for the dynamic interaction between longitudinally invariant structures and a layered halfspace. Computer Methods in Applied Mechanics and Engineering 2010; 199(23-24):1536-1548. URL http://dx.doi.org/10.1016/j.cma.2010.01.001.

52. Rayleigh J. On waves propagated along the plane surface of an elastic solid. Proceedings of the London Mathematical Society 1887; 17:4-11.

53. http://www.rivas-project.eu 2011. 